\title{
CEACAM1 negatively regulates platelet-collagen interactions and thrombus growth in vitro and in vivo
}

Wong, Cyndi; Liu, Yong; Yip, Chum Wai Jana; Chand, R; Wee, Janet; Oates, Lisa; Nieswandt, Bernhard https://researchrepository.rmit.edu.au/esploro/outputs/9921858190501341/filesAndLinks?institution=61RMIT_INST\&index=null

Wong, C., Liu, Y., Yip, C. W. J., Chand, R., Wee, J., Oates, L., Nieswandt, B., Reheman, A., Ni, H., Beauchemin, N., \& Jackson, D. (2009). CEACAM1 negatively regulates platelet-collagen interactions and thrombus growth in vitro and in vivo. Blood, 113(8), 1818-1828. https://doi.org/10.1182/blood-2008-06-165043 Document Version: Accepted Manuscript

Published Version: https://doi.org/10.1182/blood-2008-06-165043

Repository homepage: https://researchrepository.rmit.edu.au

(c) 2009 by The American Society of Hematology

Downloaded On 2023/04/26 19:36:40 +1000 
Thank you for downloading this document from the RMIT Research Repository.

The RMIT Research Repository is an open access database showcasing the research outputs of RMIT University researchers.

RMIT Research Repository: http://researchbank.rmit.edu.au/

\section{Citation:}

Wong, C, Liu, Y, Yip, C, Chand, R, Wee, J, Oates, L, Nieswandt, B, Reheman, A, Ni, H, Beauchemin, $\mathrm{N}$ and Jackson, D 2009, 'CEACAM1 negatively regulates platelet-collagen interactions and thrombus growth in vitro and in vivo', Blood, vol. 113, no. 8, pp. 1818-1828.

See this record in the RMIT Research Repository at:

https://researchbank.rmit.edu.au/view/rmit:18756

Version: Accepted Manuscript

Copyright Statement: (c) 2009 by The American Society of Hematology

Link to Published Version:

http://dx.doi.org/10.1182/blood-2008-06-165043 


\section{Revised BLOOD/2008/165043 R2 Wong et al., CEACAM1 negatively regulates platelet-collagen interactions}

and thrombus growth in vitro and in vivo.

By Cyndi Wong, ${ }^{1,5}$ Yong Liu, ${ }^{1,5}$ Jana Yip, ${ }^{1}$ Rochna Chand, ${ }^{1}$ Janet L. Wee, ${ }^{1}$ Lisa Oates, ${ }^{1}$ Bernhard Nieswandt ${ }^{2}$, Adili Reheman ${ }^{3}$, Heyu Ni, ${ }^{3}$ Nicole Beauchemin, ${ }^{4}$ and Denise E. Jackson, 1,6

From ${ }^{1}$ Kronheimer Building, Burnet Institute incorporating the Austin Research Institute, Heidelberg, Victoria, Australia, ${ }^{2}$ Rudolf Virchow Center for Experimental Biomedicine, University of Wurzburg, Germany ${ }^{3,}$ Department of Laboratory Medicine and Pathobiology, St. Michael's Hospital, University of Toronto and Canadian Blood Services, Toronto, Ontario, Canada, ${ }^{4} \mathrm{McGill}$ Cancer Centre, McGill University, Montreal, Quebec, Canada.

${ }^{5}$ Denotes both authors made an equal contribution to this manuscript.

${ }^{6} \mathrm{DEJ}$ is a recipient of an NHMRC Senior Research Fellowship.

Running Title: CEACAM1 modulates platelet-collagen interactions.

Scientific Heading: Hemostasis, Thrombosis and Vascular Biology.

This work was supported by grants from the National Heart Foundation of Australia and

National Health and Medical Research Council of Australia (to DEJ) and the Canadian

Institutes of Health Research (to NB).

Word Count: 5511. Abstract: 204.

Address correspondence to:

Assoc. Prof. Denise E. Jackson, FAIMS, PhD

NHMRC Senior Research Fellow,

Kronheimer Building,

Burnet Institute incorporating the Austin Research Institute, Studley Road, Heidelberg. Victoria. 3084.

Australia.

Phone: 613 9287-0657; Fax: 613 9287-0600

E-mail:djackson@burnet.edu.au 


\begin{abstract}
Carcinoembryonic antigen cell adhesion molecule-1 (CEACAM1) is a surface glycoprotein expressed on various blood cells, epithelial cells and vascular cells. CEACAM1 possesses adhesive and signaling properties mediated by its intrinsic immunoreceptor tyrosine-based inhibitory motifs that recruits SHP-1 protein-tyrosine phosphatase. In this study, we demonstrate that CEACAM1 is expressed on the surface and in intracellular pools of platelets. In addition, CEACAM1 serves to negatively regulate platelet collagen GPVI-FcR gamma chain signaling. Ceacam ${ }^{-/}$platelets displayed enhanced Type I collagen and GPVI-selective ligand, collagen-related peptide, CRP-mediated platelet aggregation, enhanced platelet adhesion on Type I collagen and elevated CRP-mediated alpha and dense granule secretion. Platelets derived from Ceacam $^{-/-}$mice form larger thrombi when perfused over a collagen matrix under arterial flow compared to wild-type mice. Furthermore, using intravital microscopy to ferric chloride-injured mesenteric arterioles, we show that thrombi formed in Ceacam1 ${ }^{-/}$mice were larger and were more stable than wild-type mice in vivo. GPVI depletion using monoclonal antibody JAQ1 treatment of Ceacam $1^{-/-}$mice showed a reversal in the more stable thrombus growth phenotype. Ceacam ${ }^{-/}$mice were more susceptible Type I collagen induced pulmonary thromboembolism than wild-type mice. Thus, CEACAM1 acts as a negative regulator of platelet-collagen interactions and thrombus growth involving collagen GPVI receptor in vitro and in vivo.
\end{abstract}




\section{Introduction}

In vivo, several negative regulatory mechanisms have been described that down modulate platelet-collagen interactions. These include prostacylin and nitric oxide released from endothelium and the immunoglobulin (Ig)-immunoreceptor tyrosine-based inhibitory motif (ITIM) superfamily member, PECAM-1 that serves an autoregulatory role when

platelets come into contact with each other after exposure to collagen. ${ }^{1-4}$ Given the importance of collagen in promoting platelet adhesion and thrombus formation particularly involving GPVI/FcR gamma chain signaling, it is essential that natural inhibitors that contribute to autoregulatory mechanisms that modulate collagen GPVI/FcR gamma chain-mediated signaling are characterized in platelets.

While the ITIM -bearing receptor regulation of ITAM-associated pathways has been well described in the leukocyte world, they are poorly defined in platelets. This concept is further complicated by the observation that Ig-ITIM-bearing receptors have the capacity to bind multiple phosphatases including SHP-1, SHP-2 and SHIP to initiate signaling attenuation. $^{5}$ However, there is also evidence that Ig-ITIM bearing receptors may differentially associate with distinct phosphatases depending upon the cellular context and activational status of cells. ${ }^{6,7}$

These inhibitory mechanisms are further complicated by the fact that ITAM-bearing receptors can mediate inhibitory ITAM signaling under defined conditions using either DAP12 adaptor or FcR gamma chain. ${ }^{8,9}$ This switch between activating and inhibitory ITAM signaling is controlled by the avidity of the ligand cross-linking the receptor. It 
appears that low-avidity ligand interactions result in inhibitory signaling possibly involving a phosphatase and high-avidity ligand interactions produce activating ITAMmediated signaling. ${ }^{8,9}$ Therefore, it is important to dissect out the respective mechanisms of activation and inhibitory mechanisms in platelets.

As platelets lack the prototypic inhibitory Ig-ITIM superfamily member, FcgammaRIIb, there has been a prevailing view that PECAM-1 represents the sole surrogate Ig-ITIM superfamily member that serves a negative regulatory role. ${ }^{1,2}$ However, based upon several studies it would appear that platelets contain multiple Ig-ITIM superfamily members that are likely to have a modulatory role in regulating platelet-collagen interactions. Several reports suggest that platelets contain other Ig-ITIM superfamily members including G6B and TREM (TLT-1) that may attenuate platelet function. ${ }^{10,11}$ In immunological systems, SHP-1 protein-tyrosine phosphatase is classified as a negative regulator, while in collagen GPVI platelet responses, it has been proposed to act as a positive regulator. ${ }^{12}$ Given this controversy, it is important that we study respective IgITIM superfamily members that are linked to distinct protein-tyrosine phosphatases such as SHP-1 in platelets to determine their relative importance in regulating platelet thrombus formation.

Carcinoembryonic antigen-related cell âahesion molecule 1 (CEACAM1/CD66a/Bgp) is a member of the CEACAM family of genes that is a type I-transmembrane receptor that is broadly expressed on a wide range of cells including immune, haematological, epithelial and endothelial cells. ${ }^{13}$ CEACAM1 is the only CEACAM superfamily member 
expressed in rodents and humans. It shares some similarities to PECAM-1 in its capacity to mediate homophilic and heterophilic ligands (CEA and CEACAM6) but generally has a preference towards homophilic CEACAM1 interactions. ${ }^{13}$ CEACAM1 is composed of an $\mathrm{N}$-terminal $\mathrm{IgV}$-like domain that is responsible for homophilic adhesion, followed by one to three IgC-like domains in its extracellular structure. In addition, alternative mRNA splicing gives rise to different isoforms that differ in length of their cytoplasmic domain including the long ITIM-containing isoform (CEACAM1-L)(70-73 amino acids) or a short ITIM-less isoform (CEACAM1-S) (10-12 amino acids). ${ }^{13}$ The long form of CEACAM1 protein has two ITIMs that preferentially recruit SHP-1 protein-tyrosine phosphatase and to a lesser extent SHP-2 protein-tyrosine phosphatase. ${ }^{14,15}$ Based upon recent studies in T-cells, CEACAM1 functions as a negative regulator via its recruitment and activation of SHP-1 protein-tyrosine phosphatase. ${ }^{16}$ However, there is little known about the presence and functional role of CEACAM1 in regulating platelet-collagen interactions and thrombus formation in vitro and in vivo. The only exception was a report by Hansson et al., 1990 which suggested that C-CAM (old terminology) was present in the intracellular compartment of rat platelets. ${ }^{17}$

In this current study, we provide the first report that CEACAM1 serves as a negative regulator of platelet-collagen interactions, thrombus growth in vitro and in vivo and susceptibility to Type I collagen-induced pulmonary thromboembolism. Based upon these studies, we propose CEACAM1 as a new negative regulator in platelets that serves an autoregulatory role when platelets come into contact with each other after exposure to collagen. 


\section{Materials and Methods}

\section{Materials}

\section{Antibodies}

Rabbit anti-mouse CEACAM1 has been previously described. ${ }^{18}$ Anti-rabbit FITC was purchased from Dako Australia (Glostrup, Denmark). Anti-mouse GPVI ${ }^{19}$, anti-mouse GPIb $\alpha / \mathrm{IX} / \mathrm{V}$ complex $^{20}$, anti-mouse integrin $\alpha 2 \beta 1^{21}$, anti-mouse CD9 ${ }^{22}$ anti-mouse GPVI $(\mathrm{JAQ} 1)^{23}$ were purchased or obtained from Emfret analytics (Wurzburg, Germany). Anti-mouse integrin $\alpha \operatorname{IIb} \beta 3^{24}$, anti-mouse $\mathrm{CD}_{4} 4^{25}$ and HRP-conjugated antiphosphotyrosine RC20 antibody were obtained from BD Biosciences Pharmingen (Franklin Lakes, NJ). Anti-mouse '390’ PECAM-1 antibody was provided by Dr. Steve Albelda (Philadelphia, PA). ${ }^{26}$ PLC $\gamma 2$ and ERK-2 antibodies were purchased from Santa Cruz (Santa Cruz, CA).

\section{Mice}

The generation of Ceacam1 $1^{-/}$mice and breeding on C57BL/6 background has been described. ${ }^{18}$ Wild-type and Ceacaml ${ }^{+/}$mice were age- and sex-matched C57BL/6. These mice were housed in the Burnet Institute at Austin Animal House and the St. Michael's Hospital Research Vivarium. All procedures were approved by the Austin Health Animal Ethics committee \#2005:2098 and \#2005:02354, and Animal Care Committee of St. Michael's Hospital.

\section{Preparation of mouse platelets}


Platelet-rich plasma (PRP) or washed mouse platelets were prepared as previously described. $^{26}$

\section{Static platelet adhesion assays}

Static platelet adhesion assays were performed according to Yuan et al., $1999 .{ }^{27}$ Washed platelets derived from wild-type, Ceacaml ${ }^{+/}$and Ceacaml $^{-/-}$mice were examined for binding to immobilised Type I fibrillar collagen and fibrinogen in the absence of $\mathrm{Mg}^{2+}$ over time, fixed and quantitated by differential interference contrast (DIC) microscopy (x63 oil objective).

\section{Platelet adhesion and thrombus formation under flow}

Platelet adhesion and thrombus formation under flow was performed as previously described. ${ }^{1}$

\section{Platelet aggregation assays}

Platelet aggregation assays were monitored by measuring light transmission using a 4channel platelet aggregometer (Chronolog, Havertown, PA). ${ }^{27,29}$ Platelet rich plasma (PRP) was diluted in RCD buffer, $\mathrm{pH} 7.4$, (108 mM NaCl, $38 \mathrm{mM} \mathrm{KCl}, 1.7 \mathrm{mM}$ $\mathrm{NaHCO}_{3}, 21.2 \mathrm{mM}$ sodium citrate, $27.8 \mathrm{mM}$ glucose and $1.1 \mathrm{mM} \mathrm{MgCl} 2.6 \mathrm{H}_{2} \mathrm{O}$ ) to generate a platelet count of $100 \times 10^{9} / \mathrm{L}$. Reactions were performed in glass cuvettes in a $250 \mu \mathrm{L}$ volume in the presence of $100 \mu \mathrm{g} / \mathrm{ml}$ fibrinogen and $1 \mathrm{mM} \mathrm{CaCl}_{2}$ at $37^{\circ} \mathrm{C}$ with constant stirring (1000 rpm). 


\section{Flow cytometry}

The surface and intracellular expression of CEACAM1 was performed essentially as previously described. ${ }^{30}$ The only exception was that platelets were labelled with either preimmune rabbit serum (1:500), rabbit anti-mouse CEACAM1 2457 serum (1:500) or FITC-conjugated antibody to P-selectin $(10 \mu \mathrm{g} / \mathrm{mL})$ and where appropriate then labelled with swine anti-rabbit $\mathrm{F}(\mathrm{ab})_{2}$ FITC-conjugated antibody (1:200)(Dako Australia, Glostrup, Denmark).

\section{5-Hydroxytryptamine dense granule secretion}

Dense granule secretion was performed essentially as described with the inclusion of 2 mM Imipramine hydrochloride (Sigma Chemical Co., St. Louis, MO). ${ }^{1}$

\section{Ferric chloride-induced model of thrombosis and intravital microscopy}

Wild-type, Ceacam1 $1^{+/}$or Ceacam1 ${ }^{-/}$C57BL/6 mice (4-5 weeks old) were anaesthetized with a ketamine/xylazine $(200: 10 \mathrm{mg} / \mathrm{kg})$ mixture and the mesentery was exteriorized through a midline abdominal incision. A catheter was inserted into the jugular vein to deliver rhodamine dye and anaesthetic as required. Mesenteric arterioles $(60-100 \mu \mathrm{m}$ in diameter) were visualized with a Zeiss Axiovert 135 M1 microscope (Carl Zeiss, Gottingen, Germany), and z-stack slices of the vessel captured with a AxioCam MRm camera. A strip of filter paper (1 x $4 \mathrm{~mm})$ was dipped in a $462 \mathrm{mM}\left(7.5 \%\right.$ w/v) $\mathrm{FeCl}_{3}$ solution for 3 seconds, applied to a $2-5 \mathrm{~mm}$ length of arteriole for 4 minutes and then removed. $^{30,31}$ Z-stack images of the vessel were taken over five 2 minute cycles (determined as the time the vessel begins to occlude). The rendered z-stack images were deconvolved with AxioVision Rel 4.6 software, to produce a three-dimensional image, and the volume of the thrombi were determined from the area and height of the clot. Assessment of platelet thrombi was blinded to detect quantitative differences between wild-type, Ceacam1 ${ }^{+/-}$and Ceacam1 $1^{-/}$mice. Stability was determined by calculating the 
percentage of the vessel that is occupied by the thrombus over a period of 2 minutes, and scored from $1-10$, with 1 being $0-10 \%$ occupancy and 10 being $91-100 \%$ occupancy (i.e. complete vessel occlusion).

\section{Pulmonary Thromboembolism mouse model}

Mouse models of pulmonary thromboembolism were essentially performed as previously described. ${ }^{32}$ The only exceptions include that $350 \mu \mathrm{g} / \mathrm{kg}$ of type I fibrillar collagen and $0.200 \mu \mathrm{l} / \mathrm{g}$ tissue thromboplastin (Thromborel S) were used in respective models. The endpoint of the pulmonary thromboembolism was defined as the time to restrained breathing within 10 second intervals.

\section{Statistical analysis}

Data was checked for normal distribution using the Shapiro-Wilk normality test and statistical significance was determined using unpaired Student's $t$ test. P values of 0.05 or less were taken to indicate significant differences. 


\section{Results}

\section{CEACAM1-deficient mice have normal haematopoiesis}

Unlike other CEACAM1 family members, CEACAM1 is broadly expressed on a wide range of cells including immune, epithelial cells, endothelial cells and platelets. ${ }^{13}$ In order to test if deletion of CEACAM1 influenced haematopoiesis and particularly platelet production, we determined peripheral blood haematological parameters on age- and sex matched wild-type versus CEACAM1-deficient mice. As shown in Table 1, Ceacam1 ${ }^{-/-}$ mice displayed normal white blood cell (WBC) count, red blood cell (RBC) count and parameters including haemoglobin and platelet count compared to wild-type C57BL/6 mice $(\mathrm{P}>0.05, \mathrm{n}=18)$. Differential counting of white blood cells revealed that Ceacaml $^{-/-}$ mice had an increased percentage of neutrophils compared to wild-type mice $(* \mathrm{P}<0.05$, $\mathrm{n}=18)$.

CEACAM1 is expressed on the surface and in intracellular pools in murine and human platelets

The only evidence in the literature in relation to CEACAM1 in platelets was the demonstration that C-CAM (old terminology) was present in the intracellular compartment of rat platelets. ${ }^{17}$ In order to determine the presence of CEACAM1 protein expressed on the surface and intracellular pools of resting murine and human platelets, and if this expression is upregulated by intracellular granule release, we examined the binding of specific polyclonal anti-murine CEACAM1 antibody to either resting and thrombin-stimulated wild-type murine and human platelets, monitored by flow cytometry. In order to characterize the surface expression of CEACAM1 in murine 
platelets, washed platelets were incubated with either normal rabbit pre-immune serum (1/500 dilution) or polyclonal anti-mouse CEACAM1 antibody (1/500 dilution) followed by FITC-labelled anti-rabbit conjugate (1/200 dilution) and then analyzed by flow cytometry. In addition, characterization of the total distribution of CEACAM1 in murine platelets was determined by permeabilization of the platelet membrane with $0.1 \%(\mathrm{w} / \mathrm{v})$ saponin treatment. As shown in Figure 1A and C, a two-fold increase in the mean fluorescence intensity was observed in resting murine platelets using a specific polyclonal anti-CEACAM1 antibody compared to normal rabbit pre-immune serum (1/500 dilution) $(15.24 \pm 1.61 \square ;$ vs $7.74 \pm 1.71 ; \mathbf{\square} ; \mathrm{n}=3$, ** $\mathrm{P}<0.005)$, indicating the presence of CEACAM1 on the surface of murine platelets. In addition, a four-fold increase in the mean fluorescence intensity (MFI) was observed in saponin-treated murine platelets using a specific polyclonal anti-CEACAM1 antibody compared to normal rabbit pre-immune serum (1/500 dilution) (43.64 $\pm 8.84 \square$; vs $11.99 \pm 2.75, \mathbf{\square}, \mathrm{n}=3$, $* \mathrm{P}<0.05)$, indicating the presence of CEACAM1 in an intracellular distribution in murine platelets. As shown in Figure 1B and D, a seven-fold increase in the MFI was observed in resting human platelets using a specific monoclonal anti-CEACAM1 antibody compared to an isotype control antibody $(35.3 \pm 1.3 \square$, vs $4.0 \pm 1.2 ; \square, * * \mathrm{P}<0.005, \mathrm{n}=3)$. In addition, a four-fold increase in the MFI was observed in saponin-treated human platelets using a specific monoclonal anti-CEACAM1 antibody compared to an isotype control antibody $(50.25 \pm 17.4 \square$, vs $12.05 \pm 0.35 \square$, $* \mathrm{P}<0.05, \mathrm{n}=3)$. Cell surface expression of CEACAM1 was increased upon agonist stimulation with different doses of thrombin compared to resting murine platelets and normal pre-immune rabbit serum $(24.08 \pm 2.3 \square$, vs $15.25 \pm 0.93 \square, * \mathrm{P}<0.05, \mathrm{n}=3 ; 8.41 \pm 0.37$ ш)(Figure 1E). A similar trend was observed 
with cell surface expression of CEACAM1 following agonist stimulation with different doses of thrombin compared to resting human platelets and negative isotype antibody $(58.93 \pm 7.11 \square$, vs $31.63 \pm 2.59 \square, * \mathrm{P}<0.05, \mathrm{n}=3 ; 10.23 \pm 1.15$ ш)(Figure 1F). At higher concentrations of thrombin, a modest reduction in CEACAM1 surface expression is observed. Ceacam1 ${ }^{+/-}$platelets had $60 \%$ ceacam1 expression compared to wild-type murine platelets (Figure 1G-H). These results indicate that CEACAM1 is expressed on the surface and in intracellular pools of resting murine and human platelets.

CEACAM1 serves as a negative regulator of collagen GPVI-mediated platelet responses

Recent studies in T-cells have demonstrated that CEACAM1 acts as an inhibitory coreceptor via the interface of its intrinsic ITIMs with recruitment and activation of SHP-1 protein-tyrosine phosphatase. ${ }^{16}$ This finding supports the concept in immunological systems that SHP-1 protein-tyrosine phosphatase acts as a negative regulator. ${ }^{33}$ However, in collagen GPVI-mediated platelet responses, SHP-1 has been proposed to act as a positive regulator. ${ }^{34}$ Given this controversy, it is important to define the functional role of CEACAM1 negative regulatory signaling in platelets. In order to test the hypothesis that CEACAM1, like PECAM-1, normally functions to modulate collagen GPVImediated platelet responses, we first examined the ability of platelets derived from wildtype versus CEACAM1-deficient mice to bind to immobilised Type I fibrillar collagen and fibrinogen in the absence of $\mathrm{Mg}^{2+}$ over time. As shown in Figure 2A, Ceacaml ${ }^{-/-}$ platelets bound significantly better at all time points on Type I fibrillar collagen compared to wild-type and Ceacam1 ${ }^{+/}$platelets $(* * * \mathrm{P}<0.005, \mathrm{n}=3)$. This effect was 
specific as adhesion of bovine serum albumin over the same time points was insignificant for both wild-type, Ceacaml ${ }^{+/}$and Ceacam1 ${ }^{-/}$platelets. This hyper-responsive platelet adhesion observed with Ceacam1 $1^{-/}$platelets was selective for Type I fibrillar collagen as platelet adhesion on fibrinogen did not show increased adhesion (Fig. 2B). In fact, a mild reduction in platelet adhesion on fibrinogen is observed in Ceacam1 ${ }^{-/}$platelets but not wild-type or Ceacam1 ${ }^{+/-}$platelets $(* \mathrm{P}<0.05, \mathrm{n}=3)$.

In order to examine the functional role of CEACAM1 negative regulatory signaling in platelets, we performed a time course (0-3 min) of CRP stimulation of wild-type and Ceacam $^{-/}$platelets and determined global tyrosine phosphorylation profiles of $30 \mu \mathrm{g}$ of platelet lysates. As shown in Figure 3A, CRP stimulation of Ceacam1 ${ }^{\text {/- }}$ platelets showed an increased level of tyrosine phosphorylation of a number of proteins including Syk over time with both quantitative and kinetic differences than wild-type platelets. In addition, Erk-2 blot was included as a loading control (lower panel). Measurement of tyrosine phosphorylation of PLC $\gamma 2$ was performed by immunoprecipitation of resting versus CRP stimulated wild-type and Ceacam1 $1^{-/}$platelets ( $\mathrm{T}=90$ secs) followed by immunoblotting with an anti-phosphotyrosine antibody (Fig. 3B). PLC $\gamma 2$ antigen loading was confirmed by reprobing with a polyclonal anti-PLC $\gamma 2$ antibody. As shown in Fig. 3B, resting and CRP stimulated Ceacam1 ${ }^{-/}$platelets showed increased tyrosine phosphorylation of PLC $\gamma 2$ compared to wild-type platelets. These results support the concept that CEACAM1 acts as a negative regulator to modulate ITAM-coupled signaling pathways. 
As CEACAM1 has been demonstrated to function as an regulator in T cells upon TCR ligation, we wanted to test the hypothesis that CEACAM1 serves to negatively regulate ITAM-bearing collagen GPVI-mediated platelet responses. In order to test the possibility in platelets, a comparison of the ability of platelets derived from wild-type and CEACAM1-deficient mice in platelet aggregation responses over a dose-dependent range of GPVI selective agonist, CRP $(0.62-2.5 \mu \mathrm{g} / \mathrm{mL})(\mathrm{j}-1)$ and acid soluble Type I collagen $(1.5-4 \mu \mathrm{g} / \mathrm{mL})(\mathrm{m}-\mathrm{o})$ with G-protein coupled agonists, PAR-4 (100-250 $\mu \mathrm{M})(\mathrm{a}-\mathrm{c})$, ADP $(5-20 \mu \mathrm{M})(\mathrm{d}-\mathrm{f})$ and calcium ionophore $(\mathrm{CI})(0.3-2.5 \mu \mathrm{g} / \mathrm{mL})(\mathrm{g}-\mathrm{i})$. As shown in Figure 4, wild-type and $\mathrm{Ceacaml}^{-/-}$platelets display equivalent platelet aggregation profiles over a dose-dependent range of PAR-4, ADP and calcium ionophore. In contrast, Ceacaml $1^{-/}$ platelets show enhanced amplitude and slope of platelet aggregation responses to CRP and collagen particularly at subthreshold doses. This hyper-responsive effect is independent of GPVI expression and other platelet glycoprotein expression (integrin $\alpha 2 \beta 1$, GPIb-IX-V complex, integrin $\alpha \mathrm{IIb} \beta 3, \mathrm{CD} 9, \mathrm{CD} 44$, PECAM-1) as Ceacam1 ${ }^{-/}$ platelets are comparable to wild-type platelets (Table 2). These results indicate that the absence of the Ig-ITIM superfamily member, CEACAM1 leads to hyper-responsive GPVI-mediated platelet responses to collagen and CRP.

As Ceacam $1^{-/}$platelets displayed enhanced adhesion of Type I fibrillar collagen and hyper-responsive GPVI-mediated platelet aggregation responses, we wanted to determine if the absence of CEACAM1 influenced the platelet release reaction stimulated with GPVI-specific agonists is restricted to GPVI. In order to test this possibility, we compared the ability of wild-type, Ceacam1 $1^{+-}$and Ceacam1 $1^{-/-}$platelets to secrete alpha 
(P-selectin) and dense granule serotonin upon stimulation of GPVI. As shown in Figure 5A-B, Ceacam1 $1^{-/}$platelets displayed increased release of their alpha and dense granules at subthreshold doses of fibrillar collagen and CRP compared to low or high doses of

thrombin treated wild-type and Ceacam1 ${ }^{+/}$platelets $(* \mathrm{P}<0.05, * * * \mathrm{P}<0.001, \mathrm{n}=3)$. These data are consistent with the concept that CEACAM1 normally acts as a negative regulator of platelet GPVI-mediated alpha and dense granule release.

CEACAM1-deficient mice display increased thrombus growth in vitro, in vivo and enhanced susceptibility to Type I collagen induced pulmonary thromboembolism

While platelets lack the prototypic Ig-ITIM superfamily member, Fc $\gamma$ RIIb, other surrogate Ig-ITIM superfamily members, like PECAM-1, serve as potent negative regulators of platelet thrombus formation. It is however, not clearly defined which IgITIM superfamily members are functionally important in regulating in vivo platelet thrombus formation and this issue warrants investigation. In order to explore the possibility that CEACAM1 is important in modulation of platelet thrombus formation in vivo, we compared age- and sex-matched wild-type versus Ceacam1 $1^{-/}$mice in platelet adhesion and thrombus growth on exposed extracellular matrix of the injured mesenteric arterioles (60-100 $\mu \mathrm{m}$ sized vessels) as a vascular injury model by ferric chloridemediated denuding of endothelium monitored by fluorescence intravital microscopy. The ferric chloride $\left(\mathrm{FeCl}_{3}\right)$ oxidative injury model was used to induce formation of free radicals to disrupt the vascular endothelium in mesenteric arterioles. ${ }^{30}$ Platelet thrombus formation is monitored in real time by assessing the accumulation of fluorescently labelled platelets. Following application of $\mathrm{FeCl}_{3}$ to the mesentery, platelets in both 
wild-type and Ceacaml ${ }^{-/-}$mice rapidly commenced interacting with the injured vessel wall and within 10-15 mins after the injury, vessel occlusion occurred. Thrombus characteristics were assessed in real time measuring the area $\left(\mu \mathrm{m}^{2}\right)$, height $(\mu \mathrm{m})$ and volume $\left(\mu \mathrm{m}^{3}\right)$ occupied by the thrombus together with a stability score for each thrombus over a 2 min time frame. Typically, Ceacam $1^{-t}$-mice showed increased thrombus growth and more stable thrombi over time compared to wild-type control mice (Fig. 6A). Typically, Ceacam $1^{-\%}$ arterioles showed an increase in thrombus area (reflecting surface coverage of platelets) over a 2 minute time interval compared to wild-type and Ceacaml $^{+/-}$arterioles $\left(6212 \pm 268.7\right.$ vs $3294 \pm 223.4$ and $2859 \pm 293.9 \mu \mathrm{m}^{2} ; * * * \mathrm{P}<0.0001$, n=15)(Fig. 6B). In addition, Ceacam $1^{-1}$ mice developed thrombi that were significantly larger in volume compared to wild-type and Ceacaml $^{+/-}$mice $(122400 \pm 6794$ vs $49430 \pm 4602$ and $63680 \pm 5478 \mu \mathrm{m}^{3} ; * * * \mathrm{P}<0.0001, \mathrm{n}=15$ ) (Fig. 6C). Moreover, the thrombi formed in Ceacam $^{-/}$arterioles were typically more stable than those in wildtype and Ceacam $^{+/}$arterioles $(4.067 \pm 0.3712$ vs $2.400 \pm 0.2138$ and $1.05 \pm 0.12$; $* * * \mathrm{P}<0.001, \mathrm{n}=15$ )(Fig. 6D). In contrast, Ceacam1 ${ }^{-}$mice showed no abnormality in time to first thrombi $>20 \mu \mathrm{m}^{2}$, number of thrombi $>20 \mu \mathrm{m}^{2}$, the increase in thrombus diameter over $1 \mathrm{~min}$, thrombus height and time to vessel occlusion $(\mathrm{P}>0.05, \mathrm{n}=15)$ (data not shown). In order to directly test the contribution of collagen GPVI receptor in this thrombus growth phenotype in ceacam $1^{-/-}$arterioles, we deleted GPVI in vivo using JAQ1 treatment (100 $\mu \mathrm{g} /$ mouse) and left for 5 days before ferric chloride injury and intravital studies $^{23}$. A different cohort of ceacam $1^{-/-}$were similarly treated with control IgG (100 $\mu \mathrm{g} /$ mouse). As shown in Figure 6E-F, JAQ1 treated Ceacam ${ }^{-1}$ mice showed a 3-fold reduction in thrombus volume and 2-fold reduction in stability score compared to control 
IgG treated and untreated Ceacaml ${ }^{+/+}$and Ceacam $1^{-/}$arterioles $(\mathrm{n}=10$ arterioles from 3 mice/group; $* * * \mathrm{P}<0.001)$. Importantly, Ceacam1 $1^{-/-}$arterioles showed reversal of the more stable thrombus phenotype upon JAQ1 treatment and in thrombus growth phenotype compared to Ceacaml $1^{+/+}$arterioles. We confirmed successful clearance of GPVI after JAQ1 treatment by western blotting of platelet lysates from Ceacam1 ${ }^{-/}$ platelets (not shown). This data supports the concept that the in vivo thrombus growth phenotype in ceacam $1^{-/-}$arterioles is dependent on presence of collagen GPVI receptor.

To further investigate the role of CEACAM1 in modulating platelet thrombus formation on immobilized collagen, in vitro flow studies were performed with blood obtained from wild-type, Ceacaml $1^{+/}$and Ceacam1 $1^{-/}$mice. DiOC 6 -labelled whole blood was perfused through Type I collagen-coated microcapillary tubes at a wall shear rate of 1800 seconds $^{-}$ 1, and thrombi were imaged at 4 minutes of blood perfusion by confocal microscopy. Ceacaml $^{\text {-/ }}$ platelets $\left(136200 \pm 18640 \mu^{3}\right)$ displayed increased thrombus volume than wild-type $\left(83870 \pm 8065 \mu^{3}\right)$ or Ceacaml $^{+/-}$platelets $\left(86640 \pm 15330 \mu \mathrm{m}^{3}\right) \quad(\mathrm{P}<0.05$, $\mathrm{n}=9)$ (Fig. 6F). Collectively, these data demonstrate that CEACAM1 is required for modulation of platelet-collagen interactions in platelet thrombus growth in vitro and in vivo.

To further determine the consequences of CEACAM1 deficiency in vivo, we tested wildtype, Ceacaml $1^{+/-}$and Ceacam1 $1^{-/-}$mice in a model of pulmonary thromboembolism induced by infusion of type I fibrillar collagen or tissue thromboplastin. Typically, Ceacam $^{-/-}$mice displayed enhanced susceptibility to Type I collagen induced pulmonary 
thromboembolism than wild-type and Ceacam1 $^{+/}$mice with $>70 \%$ reduction in circulating platelet counts within 3 minutes of challenge (Figure 7A-B). Following challenge with Type I fibrillar collagen, Ceacam $1^{-/-}$mice reached maximal clinical scoring of restrained breathing earlier than wild-type and Ceacam1 $1^{+/-}$mice $(252.1 \pm 15.39$ versus $343.5 \pm 23.73$ and $327.0 \pm 28.29$ secs; $* * \mathrm{P}<0.005 ; \mathrm{n}=20)$. In this system, both wildtype, Ceacaml $1^{+/-}$and Ceacaml $1^{-/-}$mice had comparable reductions in circulating platelet numbers over a three minute time frame tested following challenge with type I fibrillar collagen $(73.49 \pm 3.15$ versus $77.24 \pm 2.25$ and $74.21 \pm 3.50 \% ; \mathrm{P}>0.05, \mathrm{n}=20)$. It should be noted that early time points could not be tested for detection of kinetic differences in platelet consumption due to animal welfare considerations. Consistent with these observations, histologic sections of lung tissue and analysis of occlusive thrombi in the lungs at the end of the experiment revealed similar numbers of occluded vessels between wild-type, Ceacaml ${ }^{+/-}$and Ceacam1 $1^{-/-}$mice (data not shown). In contrast, wild-type and Ceacam $^{-/-}$mice were equally susceptible and had comparable reductions in circulating platelets over time following tissue thromboplastin-induced pulmonary thromboembolism (Figure 7C-D) $(\mathrm{P}>0.05, \mathrm{n}=10)$. Collectively, these data highlight that CEACAM1 regulates platelet-collagen interactions in controlling susceptibility to Type I collagen-induced pulmonary thromboembolism in vivo. 


\section{Discussion}

Using several approaches, we have defined a novel role of CEACAM1 as a new inhibitory co-receptor in murine platelets that serves to negatively modulate plateletcollagen interactions and regulate platelet thrombus formation in vitro and in vivo. This study suggests that CEACAM1 serves an autoregulatory role when platelets come into contact with each other when exposed to Type I collagen. Firstly, CEACAM1 is expressed on the surface and in intracellular pools of murine and human platelets. Secondly, CEACAM1 serves as a negative regulator of collagen GPVI-mediated platelet responses in vitro. Thirdly, CEACAM1 serves to negatively regulate platelet thrombus formation in vitro and in vivo under conditions where Type I collagen is exposed in the injured arteriole and in the presence of collagen GPVI receptor on platelets. Finally, CEACAM1 serves as a negative regulator of thrombosis following Type I collageninduced pulmonary thromboembolism in vivo.

There is little evidence in the literature in relation to the presence and functional role of CEACAM1 in platelets. The only exception is a report by Hansson et al., 1990 which suggested that C-CAM (old terminology) was present in the intracellular compartment of rat platelets. ${ }^{17}$ In these studies, these workers only found significant amounts of C-CAM when ${ }^{125}$ I-labelled rat platelets were solubilised with detergent and immunoprecipitated with C-CAM antibodies. These workers suggested that almost no ${ }^{125}$ I-labelled C-CAM could be immunoprecipitated from intact rat platelets under resting or ADP-activated conditions. This study suggested that intracellular expressed C-CAM may play a functional role under conditions of platelet activation. This concept is supported by studies of CEACAM1 in leukocytes. Traditionally, CEACAM1 (formerly known as 
CD66a) was defined as a neutrophil-specific "activation antigen" in that it is detected in low density on resting cells but its surface expression is upregulated upon stimulation with the chemotactic peptide FMLP, calcium ionophore A23187 and 12-O-tetradeconoylphorbol-13-acetate. ${ }^{35}$ Subcellular localization studies revealed that CEACAM1 was present in the secondary granules of neutrophils suggesting that antigens could be recruited to the cell surface with activation. ${ }^{36}$ In contrast, in mouse spleen $\mathrm{T}$ lymphocytes, CEACAM1 was not present on the surface of resting cells but was rapidly upregulated on $\mathrm{CD}^{+}$and $\mathrm{CD}^{+} \mathrm{T}$ cells after activation with either Con A or anti-CD3 stimulation. $^{37}$ Overall, these studies in leukocytes are somewhat different from the CEACAM1 expression profile that we have observed in murine and human platelets with moderate basal expression on the surface of resting platelets with an intracellular pool evident (Fig.1A-D). This was further supported by agonist stimulation using thrombin that resulted in upregulation of cell surface expression of CEACAM1 on murine and human platelets (Fig.1E-F).

There is now a consensus view that platelet thrombus formation under physiological conditions involves platelet adhesion to subendothelial matrix component, Type I collagen which becomes exposed by tissue trauma, injury or disease such as atherosclerotic plaque formation. A 'two-site, two-step' model for platelet-collagen interactions has been proposed that requires the initial engagement of collagen to be mediated by the high-affinity receptor integrin $\alpha 2 \beta 1$, followed by activation generated through the low-affinity collagen receptor, GPVI ${ }^{38,39}$ In this model, integrin $\alpha 2 \beta 1$ is now thought to have a supportive, rather than a primary role as the adhesive collagen receptor, while GPVI is a major platelet-collagen activating receptor that interacts with collagen 
fibrils via a triplicate GPO sequence (glycine, proline, hydroxyproline) and integrin $\alpha 2 \beta 1$ interacts with collagen via GFOFER (glycine, phenylalanine, hydroxyproline, glutamic acid and arginine). ${ }^{40-42}$ For in vitro studies, we used collagen-related peptide (CRP) as the GPVI-selective agonist as this peptide contains ten GPO repeat sequences that serves as a potent activator of GPVI-mediated signaling and does not recognize integrin $\alpha 2 \beta 1^{43}$ In this way, we can differentiate GPVI-mediated platelet responses from integrin $\alpha 2 \beta 1$ mediated platelet responses. Signaling through GPVI/FcR gamma chain ITAMdependent pathway is thought to be counterbalanced by proteins bearing ITIM motifs such as those contained in members of the Ig-ITIM superfamily. As mouse platelets lack the low-affinity ITAM containing Fc $\gamma$ RIIa, but contain GPVI/FcR gamma chain ITAMdependent pathway, we wanted to test the hypothesis that CEACAM1 may serve as an inhibitory co-receptor in platelets to negatively regulate collagen GPVI-mediated platelet

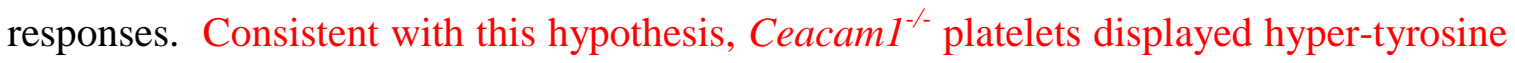
phosphorylated proteins including PLC gamma 2 following GPVI stimulation compared to wild-type platelets indicating modulation of ITAM-associated signaling events in murine platelets (Fig. 3).

In order to test this hypothesis, platelet aggregation studies were conducted to examine the slope and amplitude of platelet responses of wild-type versus CEACAM1-deficient platelets using a range of agonists and dose-dependent ranges including collagen and CRP. In addition, to test specificity of platelet responses, different agonists were selected to stimulate different receptor signaling pathways including G-protein-coupled receptors using PAR-4 and ADP, calcium ion channels using calcium ionophore and collagen 
coupled receptors such as acid soluble collagen and CRP. Ceacam1 $1^{-/-}$platelets displayed normal platelet aggregation responses over a dose-dependent range of PAR-4, ADP and calcium ionophore. In contrast, Ceacaml ${ }^{-/-}$platelets displayed enhanced platelet aggregation responses over a dose-dependent range of collagen and CRP (Fig.4). This hyper-responsive feature was most evident at subthreshold concentrations of either collagen or CRP indicating that CEACAM1 serves to negatively regulate collagen GPVImediated ITAM platelet responses. Importantly, the fact that the platelet aggregation responses to PAR-4, ADP and calcium ionophore were normal reveals that Ceacam1 ${ }^{-/-}$ platelets do not have a global platelet defect and that the defect is restricted to GPVImediated platelet-collagen interactions.

This hyper-responsive feature of Ceacaml $^{-/-}$platelets with either subthreshold doses of collagen or CRP is consistent with the phenotype observed by us and others with Pecam1 $^{-/}$platelets. $^{1,2}$ The subtle phenotype was most evident at concentrations of collagen at $1 \mu \mathrm{g} / \mathrm{mL}$ but was not so evident at higher concentrations of collagen 10-30 $\mu \mathrm{g} / \mathrm{mL}$. This was also the case using CRP as the GPVI-selective agonist. At $0.5 \mu \mathrm{g} / \mathrm{mL}$ CRP, wild-type platelets were not responsive while Pecam- $1^{-/-}$platelets yielded approx. 70\% CRP-mediated platelet aggregation response. At 5-10 $\mu \mathrm{g} / \mathrm{mL} \mathrm{CRP,} \mathrm{wild-type} \mathrm{and}$ Pecam- $1^{-/-}$platelets yielded less hyper-responsive platelet responses. ${ }^{1,2}$

Platelets from Ceacam1 ${ }^{-/}$mice exhibited increased adhesion on Type I fibrillar collagen but not fibrinogen. This hyper-responsive adhesion phenotype of Ceacaml $^{-/-}$platelets on Type I fibrillar collagen was evident at all time points. In addition, Ceacaml ${ }^{-/-}$platelets 
displayed a lowered threshold for alpha and dense granule release relative to wild-type and Ceacam1 $1^{+-}$platelets following stimulation with GPVI-selective ligand, CRP particularly at $0.1-0.5 \mu \mathrm{g} / \mathrm{mL}$ and type I collagen at $1 \mu \mathrm{g} / \mathrm{mL}$ but not G-protein-coupled receptor agonist, thrombin. Overall, these features suggest that in the absence of an inhibitory co-receptor, platelets are more responsive to stimulation by collagen agonists particularly involving collagen GPVI receptor-mediated signaling. This trend is consistent with CEACAM1 serving as a negative regulator of platelet-collagen interactions.

In order to investigate the role of CEACAM1 in thrombus formation in vitro and in vivo, we employed intravital microscopy to study thrombus formation in real time and ferric chloride-induced vascular injury of mesenteric arterioles and in vitro flow studies on immobilised Type I collagen. Using this ferric chloride-induced vascular injury model results in exposure of subendothelial collagens including Type I collagen. This enables collagen GPVI platelet interactions to occur with exposed Type I collagen. Under these conditions, we demonstrated that thrombi formed in mesenteric arterioles following ferric chloride injury in CEACAM1 knockout mice were significantly larger in area and volume reflecting increased thrombus growth than those formed in wild-type and Ceacaml $^{+/-}$mice (Fig.6B-C). These differences in thrombus size were consistent over time. In addition, thrombi formed in CEACAM1 knockout mice appear to have greater stability than wild-type and Ceacaml ${ }^{+/-}$mice (Fig.6D). In addition, this in vivo thrombus growth phenotype observed in Ceacam1 ${ }^{-/}$mice was dependent on the presence of collagen GPVI receptor on platelets (Fig. 6E-F). These data are consistent with 
CEACAM1 playing a role in negative regulation of thrombus growth in vivo in mesenteric arterioles. In addition, our studies have shown an important role for CEACAM1 in regulating thrombus formation on immobilised collagen under in vitro flow conditions (Fig.6F). As CEACAM1 is expressed on platelets, leukocytes and endothelium further studies will be required to dissect out the relative importance of platelet CEACAM1 versus endothelial CEACAM1 in regulating thrombus growth in vivo. Importantly, CEACAM1 appears to represent a second example of an Ig-ITIM superfamily member that plays an important functional role in negatively regulating stationary adhesion of platelet-collagen interactions and thrombus growth in vivo, but not tethering and rolling of platelets on the thrombogenic collagen surface.

Previous studies with PECAM-1-deficient mice in the ferric chloride induced vascular injury model of carotid arteries revealed a subtle defect in the time to $75 \%$ vessel occlusion. $^{44}$ The mean occlusion rate of Pecam- ${ }^{-/-}$mice was shorter than wild-type control mice. However, the magnitude of difference was only very modest. A more noticeable difference was observed in the kinetics of platelet thrombus formation using the laser-induced injury model that requires tissue factor generated platelet/fibrin thrombus and not Type I collagen exposure. ${ }^{44}$ Therefore, it appears that PECAM-1 and CEACAM1 both play a role in negatively regulating platelet-collagen interactions in vivo.

In order to examine the consequences of CEACAM1 deficiency in vivo in plateletcollagen interactions, we utilised a second model of Type I collagen-induced pulmonary 
thromboembolism. In this model, type I fibrillar collagen is infused intravenously and platelet thrombi are deposited in the lung architecture leading to development of pulmonary thromboembolism and restrained breathing that in wild-type mice ultimately leads to morbidity. Using this model, monitoring of the restrained breathing rate of the mice revealed that Ceacaml ${ }^{-/-}$mice have enhanced susceptibility to Type I collagen induced pulmonary thromboembolism but not tissue thromboplastin induced pulmonary thromboembolism compared to wild-type and Ceacaml ${ }^{+-}$mice (Fig.7B and 7D). Ceacam $^{-/-}$mice showed a reduction in time for restrained breathing following challenge with Type I fibrillar collagen compared to wild-type and Ceacaml ${ }^{+/}$mice (Fig.7B) despite having comparable consumption of platelets (Fig.7A). These data further implicate CEACAM1 as a physiological regulator of platelet-collagen interactions in vivo.

In conclusion, this study has defined a new inhibitory co-receptor, CEACAM1 present in murine and human platelets that is expressed on the surface and in intracellular compartments. CEACAM1 serves as a negative physiological regulator of plateletcollagen interactions as shown by its hyper-responsive features in collagen and CRPmediated platelet aggregation, increased adhesion on Type I collagen and enhanced alpha and dense granule release using GPVI-selective ligand. In vitro and in vivo, CEACAM1 serves to negatively regulate arteriolar thrombus growth and thrombosis associated with Type I collagen induced pulmonary thromboembolism. These negative regulatory properties of CEACAM1 may prove to be of physiological benefit in diseased vessels such as rupture of atherosclerotic plaques where Type I collagen is exposed. 


\section{Acknowledgements}

C.Wong, Y.Liu, J. Yip, R. Chand, J. Wee, A. Reheman and L. Oates performed research and analysed data; N. Beauchemin supplied the CEACAM1-deficient mice, B. Nieswandt supplied the JAQ1 antibody and advice for the GPVI depletion experiment, $\mathrm{H}$. Ni directed the in vitro flow experiments and DE Jackson directed the research and wrote the paper. 


\section{References}

1. Jones KL, Hughan SC, Dopheide SM, Farndale RW, Jackson SP and Jackson DE. Platelet endothelial cell adhesion molecule-1 is a negative regulator of platelet-collagen interactions. Blood 2001;98(5):1456-1463.

2. Patil S, Newman DK and Newman PJ. Platelet endothelial cell adhesion molecule-1 serves as an inhibitory receptor that modulates platelet responses to collagen. Blood 2001;97(6):1727-1732.

3. Cicmil M, Thomas JM, Leduc M, Bon C and Gibbins JM. Platelet endothelial cell adhesion molecule-1 signaling inhibits the activation of human platelets. Blood 2002;99(1):137-144.

4. Dhanjal TS, Ross EA, Auger JM, McCarty OJ, Hughes CE, Senis JA, Buckley CD and Watson SP. Minimal regulation of platelet activity by PECAM-1. Platelets 2007;18(1):56-67.

5. Pao LI, Badour K, Siminovitch KA and Neel BG. Nonreceptor protein-tyrosine phosphatases in immune cell signaling. Ann. Rev. Immunol. 2007;25:473-523.

6. Chemnitz JM, Parry RV, Nichols KE, June CH and Riley JL. SHP-1 and SHP-2 associate with immunoreceptor tyrosine-based switch motif of programmed death 1 upon 
primary human $\mathrm{T}$ cell stimulation, but only receptor ligation prevents $\mathrm{T}$ cell activation. $J$. Immunol. 2004;173:945-954.

7. Okazaki T, Maeda A, Nishimura H, Kurosaki T and Honjo T. PD-1 immunoreceptor inhibits B cell receptor-mediated signaling by recruiting src homology 2-domain containing tyrosine phosphatase 2 to phosphotyrosine. Proc. Natl. Acad. Sci. USA 2001;98:13866-13871.

8. Hamerman JA and Lanier LL. Inhibition of immune responses by ITAM-bearing receptors. Science STKE 2006;320:1-7.

9. Abram CL and Lovell CA. The expanding role for ITAM-based signaling pathways in immune cells. Science STKE 2007;377:1-6.

10. Newland SA, Macaulay IC, Floto AR, de Vet EC, Ouwehand WH, Watkins NA, Lyons PA and Campbell DR. The novel inhibitory receptor G6B is expressed on the surface of platelets and attenuates platelet function in vitro. Blood 2007;109(11):48064809.

11. Washington AV, Schubert RL, Quigley L, Disipio T, Feltz R, Cho EH and McVivar DW. A TREM family member, TLT-1, is found exclusively in the alpha granules of megakaryocytes and platelets. Blood 2004;104(4):1042-1047. 
12. Pasquet JM, Quek L, Pasquet S, Poole A, Matthews JR, Lowell C and Watson SP. Evidence for a role for SHP-1 in platelet activation by the collagen receptor glycoprotein VI. J. Biol. Chem. 2000;275(37):28526-28531.

13. Horst AK and Wagener C. CEA-related CAMs. Handbook of Experimental Pharmacology, Ed Behrens J and Nelson WJ. Springer-Verlag, Heidelberg, Germany. 2004; 165:284-323.

14. Beauchemin N, Kunath T, Robitaille J, Chow B, Turbide C, Daniels E and Veillette A. Association of biliary glycoprotein with protein tyrosine phosphatase SHP-1 in malignant colon epithelial cells. Oncogene 1997;14:783-790.

15. Huber M, Izzi L, Grondin P, Houde C, Kunath T, Veillette A and Beauchemin N. The carboxy-terminal region of biliary glycoprotein controls its tyrosine phosphorylation and association with protein-tyrosine phosphatases SHP-1 and SHP-2 in epithelial cells. J. Biol. Chem. 1999;274(1):335-344.

16. Nagaishi T, Pao L, Lin SH, Iijima H, Kaser A, Qiao SW, Chen Z, Glickman J, Najjar SM, Nakajima A, Neel BG and Blumberg RS. SHP1 phosphatase-dependent T cell inhibition by CEACAM1 adhesion molecule isoforms. Immunity 2006;25(5):769-781. 
17. Hansson $\mathrm{M}$, Odin $\mathrm{P}$, Johansson $\mathrm{S}$ and Obrink B. Comparison and functional characterization of C-CAM, glycoprotein IIb/IIIa and integrin beta 1 in rat platelets. Thromb. Res. 1990;58(1):61-73.

18. Leung N, Turbide C, Olson M, Marcus V, Jothy S and Beauchemin N. Deletion of the carcinoembryonic antigen-related cell adhesion molecule 1 (Ceacam1) gene contributes to colon tumor progression in a murine model of carcinogenesis. Oncogene 2006;25:5527-5536.

19. Nieswandt B, Bergmeier W, Schulte V, Rackebrandt K, Gessner JE and Zirngibi H. Expression and function of the mouse collagen receptor glycoprotein VI is strictly dependent on its association with the FcR gamma chain. J. Biol. Chem. 2000;275(31):23998-24002.

20. Bergmeier W, Rackebrandt K, Schroder W, Zirngibl H and Nieswandt B. Structural and functional characterization of the mouse von Willebrand factor receptor GPIb-IX with novel monoclonal antibodies. Blood 2000;95:886-893.

21. Mendrick DL, Kelly DM, duMont SS and Sandstrom DJ. Glomerular epithelial and mesangial cells differentially modulate the binding specificities of VLA-1 and VLA-2. Lab Invest. 1995;72:367-375. 
22. Maecker HT, Todd SC and Levy S. The tetraspanin superfamily: molecular facilitators. FASEB J. 1997;11(6):428-442.

23. Massberg S, Gawaz M, Gruner S, Schulte V, Konrak I, Zohlnhofer D, Heinzmann U and Neiswandt B. A crucial role of glycoprotein VI for platelet recruitment to the injured arterial wall in vivo. J. Exp. Med. 2003;197(1):41-49.

24. Yasuda M, Hasunuma Y, Adachi H, Sekine C, Sakanishi T, Hashimoto H, Ra C, Yagita $\mathrm{H}$ and Okumura $\mathrm{K}$. Expression and function of fibronectin binding integrins on rat mast cells. Int. Immunol. 1995;7:251-258.

25. Trowbridge IS, Lesley H, Schulte R, Hyman R and Trotter J. Biochemical characterization and cellular distribution of a polymorphic murine cell-surface glycoprotein expressed on lymphoid tissues. Immunogenetics 1982;15:299-312.

26. Yan HC, Pilewski JM, Zhang Q, DeLisser HM, Romer L and Albelda SM. Localization of multiple functional domains on human PECAM-1 (CD31) by monoclonal antibody epitope mapping. Cell Adhes Commun. 1995;3(1):45-66.

27. Wee JL and Jackson DE. The Ig-ITIM superfamily member of PECAM-1 regulates the "outside-in" signaling properties of integrin alphaIIbbeta3 in platelets. Blood 2005;106(12):3816-3823. 
28. Yuan Y, Kulkarni S, Ulsemer P, Cramer SL, Yap CL, Nesbitt WS, Harper I, Mistry N, Dopheide SM, Hughan SC, Williamson D, de la Salle C, Salem HH, Lanza F and Jackson SP. The von Willebrand factor-glycoprotein Ib/IX/V interaction induces actin polymerization and cytoskeletal reorganization in rolling platelets and glycoprotein Ib/V/IX-transfected cells. J. Biol. Chem. 1999;274(51):36241-36251.

29. Jackson DE, Ward CM, Wang R and Newman PJ. The Protein-tyrosine phosphatase SHP-2 binds platelet/endothelial cell adhesion molecule-1 (PECAM-1) and forms a distinct signaling complex during platelet aggregation. J. Biol. Chem. 1997;272(11):6986-6993.

30. Goschnick MW, Lau LM, Wee JL, Liu YS, Hogarth PM, Robb LM, Hickey MJ, Wright MD and Jackson DE. Impaired 'outside-in' integrin alphaIIbbeta3 signaling and thrombus stability in TSSC6-deficient mice. Blood 2006;108(6):1911-1918.

31. Ni H, Denis CV, Subbarao S, Degen JL, Sato TN, Hynes RO and Wagner DD. Persistence of platelet thrombus formation in arterioles of mice lacking both von Willebrand factor and fibrinogen. J. Clin. Invest. 2000;106(3):385-392.

32. He L, Pappan DG, Grenache DG, Li Z, Tollefsen DM, Santoro SA and Zutter MM. The contribution of the $\alpha 2 \beta 1$ integrin to vascular thrombosis in vivo. Blood 2003;102:3652-3657. 
33. Tsui HW, Siminovitch KA, de Souza L and Tsui FW. Motheaten and viable motheaten mice have mutations in the haematopoietic cell phosphatase gene. Nat. Genet. $1993 ; 4(2): 124-129$.

34. Pasquet JM, Quek L, Pasquet S, Poole A, Matthews JR, Lowell C and Watson SP. Evidence for a role for SHP-1 in platelet activation by the collagen receptor glycoprotein VI. J. Biol. Chem. 2000;275(37):28256-28531.

35. Skubitz KM, Ducker TP and Goueli SA. CD66 monoclonal antibodies recognise a phosphotyrosine-containing protein bearing a carcinoembryonic antigen cross-reacting antigen on the surface of human neutrophils. Journal of Immunology 1992;148(3):852860.

36. Ducker TP and Skubitz KM. Subcellular localization of CD66, CD67, and NCA in human neutrophils. Journal of Leukocyte Biology 1992;52(1):11-16.

37. Nakajima A, Iijima H, Neurath MF, Nagaishi T, Nieuwenhuis EE, Raychowdhury R, Glickman J, Blau DM, Russell S, Holmes KV, and Blumberg RS. Activation-induced expression of carcinoembryonic antigen-cell adhesion molecule 1 regulates mouse $\mathrm{T}$ lymphocyte function. Journal of Immunology 2002;168:1028-1035. 
38. Santoro SA, Walsh JJ, Staatz WD and Baranski KJ. Distinct determinants on collagen support alpha2 beta1 integrin-mediated platelet adhesion and platelet activation. Cell Regulation 1991;2(11):905-913.

39. Morton LF, Hargreaves PG, Farndale RW, Young RD and Barnes MJ. Integrin alpha 2 beta 1 -independent activation of platelets by simple collagen-like peptides: collagen tertiary (triple-helical) and quarternary (polymeric) structures are sufficient alone for alpha 2 beta 1-independent platelet reactivity. Biochem J. 1995;306(2):337344.

40. Nieswandt B and Watson SP. Platelet-collagen interaction: is GPVI the central receptor? Blood 2003;102(2):449-461.

41. Nieswandt B, Brakebusch C, Bergmeier W, Schulte V, Bouvard D, Mokhtari-Nejad R, Lindhout T, Hemmsverk JW, Zirngibil H and Fassier R. Glycoprotein VI but not alpha2beta1 integrin is essential for platelet interaction with collagen. EMBO Journal 2001;20(9):2120-2130.

42. Chen H, Locke D, Liu Y, Liu C and Kahn ML. The platelet receptor GPVI mediates both adhesion and signaling responses to collagen in a receptor density-dependent fashion. Journal of Biological Chemistry 2002;277(4):3011-3019. 
43. Kato K, Kanaji T, Russell S, Kunicki TJ, Furihata K, Kanaji S, Marchese P, Reininger A, Ruggeri ZM and Ware J. The contribution of glycoprotein VI to stable platelet adhesion and thrombus formation illustrated by targeted gene deletion. Blood 2003;102(5):1701-1707.

44. Falati S, Patil S, Gross PL, Stapleton M, Merrill-Skoloff G, Barrett NE, Pixton KL, Weiler H, Cooley B, Newman DK, Newman PJ, Furie BC, Furie B and Gibbins JM. Platelet PECAM-1 inhibits thrombus formation in vivo. Blood 2006;107:535-541. 


\section{Figure Legends}

Figure 1. CEACAM1 is expressed on the surface and in intracellular pools in murine and human platelets.

A. Flow cytometric analysis of CEACAM1 surface and total expression on resting murine platelets. Platelets were stained with a polyclonal anti-murine CEACAM1 2457 antibody followed by a secondary FITC-conjugated anti-rabbit antibody. Normal rabbit serum and $\mathrm{Ceacaml}^{\text {/- }}$ platelets were included as negative controls. Data was collected through a live platelet gate based on forward versus side scatter profiles on a FACS Canto flow cytometer. Results are cumulative data derived from three independent experiments and represented as mean fluorescence intensity (MFI) \pm SEM.

B. Flow cytometric analysis of CEACAM1 surface and total expression on resting human platelets. Platelets were stained with a monoclonal anti-human CEACAM1 4D1C2 antibody followed by a secondary FITC-conjugated anti-mouse antibody. Normal mouse $\mathrm{IgG}$ and Ceacaml ${ }^{-/-}$platelets were included as negative controls. Data was collected through a live platelet gate based on forward versus side scatter profiles on a FACS Canto flow cytometer. Results are cumulative data derived from three independent experiments and represented as mean fluorescence intensity (MFI) \pm SEM.

C-D. Representative histogram profiles of CEACAM1 surface and total expression on resting murine and human platelets. CEACAM1 surface and total expression was determined as described in A and B.

E. Agonist stimulation of murine platelets using thrombin $(0.25-1.00 \mathrm{U} / \mathrm{mL})$ over a dosedependent range. CEACAM1 surface expression was determined as described in A. 
F. Agonist stimulation of human platelets using thrombin $(0.25-1.00 \mathrm{U} / \mathrm{mL})$ over a dosedependent range. CEACAM1 surface expression was determined as described in B.

G. Flow cytometric analysis of CEACAM1 surface expression on resting murine platelets from wild-type versus Ceacaml $^{+/}$and Ceacam $1^{-/-}$mice. CEACAM1 surface expression was determined as described in A.

H. Representative histogram profiles of CEACAM1 surface expression on resting murine platelets from wild-type versus Ceacaml ${ }^{+/-}$and Ceacaml $^{-/}$mice.

\section{Figure 2. Ceacam $1^{-/-}$platelets demonstrate increased adhesion on immobilised Type}

\section{I fibrillar collagen.}

A. Wild-type, Ceacam ${ }^{+/}$and Ceacaml ${ }^{-/-}$platelets were allowed to adhere to type I fibrillar collagen $(50 \mu \mathrm{g} / \mathrm{mL})$ in the absence of magnesium, or to plates coated with bovine serum albumin $(50 \mu \mathrm{g} / \mathrm{mL})$ for $15,30,45$ or 60 minutes at $37^{\circ} \mathrm{C}$. After removal of non-adherent platelets, adherent platelets were measured as described in Materials and Methods. Each assay was performed in triplicate and is representative of three independent experiments. Each data point represents platelet adhesion per high powered field and was expressed as the mean \pm SEM. Note that wild-type, Ceacam1 ${ }^{+/}$and Ceacam $^{-/-}$platelets bound to a similar extent on BSA-coated platelets, where at all time points, Ceacam1 $1^{-/}$platelets showed higher levels of adhesion to type I fibrillar collagen compared to wild-type and Ceacaml $^{+-}$platelets $(* * * \mathrm{P}<0.005, \mathrm{n}=3)$. B. As described in A, but for platelet adhesion on fibrinogen $(50 \mu \mathrm{g} / \mathrm{mL})$ for wild-type, Ceacaml ${ }^{+/}$and Ceacam1 $1^{-/-}$platelets for $15,30,45$ or 60 minutes at $37^{\circ} \mathrm{C}$. 
C. Representative micrographs of platelet adhesion on Type I fibrillar collagen (50 $\mu \mathrm{g} / \mathrm{mL}$ ) for wild-type and Ceacam $1^{-/}$platelets over time. Adherent platelets were fixed and imaged for DIC microscopy (x 63 oil objective).

\section{Figure 3. Ceacam ${ }^{-/}$platelets display hyper-phosphorylated proteins including PLC-gamma2 following CRP stimulation over time.}

A. Tyrosine phosphorylation in whole-cell platelet lysates from wild-type and Ceacaml ${ }^{-}$ ${ }^{\prime}$ mice were stimulated with CRP $(10 \mu \mathrm{g} / \mathrm{mL})$ over a $3 \mathrm{~min}$ time frame. $30 \mu \mathrm{g}$ of each platelet lysate was loaded onto a 10\% SDS-PAGE gel and tyrosine phosphorylation detected on the western blot using a HRP-conjugated anti-phosphotyrosine RC20 antibody. Erk-2 blot (lower panel) was included as a protein loading control. This is a representative blot of three experiments performed. B. PLC $\gamma 2$ was immunoprecipitated from lysates of wild-type or Ceacam $1^{-}$platelets stimulated by CRP $(10 \mu \mathrm{g} / \mathrm{mL})$ at $\mathrm{T}=0$ and $\mathrm{T}=90$ seconds and immunoblotted for phosphotyrosine using a HRP-conjugated antiphosphotyrosine RC20 antibody. PLC $\gamma 2$ antigen is included as a loading control of the immunoprecipitates (lower panel). Relative intensity of tyrosine phosphorylated PLC $\gamma 2$ to total PLC $\gamma 2$ antigen was quantitated using Image $\mathrm{J}$ software version $1.40 \mathrm{~g}$ from three replicate experiments.

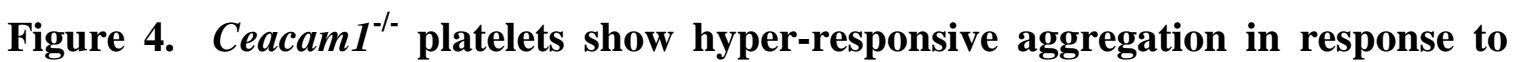
stimulation with GPVI-selective agonists.

Aggregation responses of PRP (platelet count adjusted to $100 \times 10^{9} / 1$ ) for wild-type and Ceacam $^{-1-}$ mice were determined following activation with different concentrations of 
various agonists: PAR-4 agonist peptide $(100-250 \mu \mathrm{M})$, ADP $(5-20 \mu \mathrm{M})$, calcium ionophore $(0.3-2.5 \mu \mathrm{g} / \mathrm{mL})$, collagen-related peptide $(0.62-2.5 \mu \mathrm{g} / \mathrm{mL})$ and collagen (1.5$4 \mu \mathrm{g} / \mathrm{mL}$ ) respectively. Note that Ceacam $^{-/-}$platelets are hyper-responsive in collagen and CRP-induced platelet aggregation. These experiments are representative of at least three independent experiments performed.

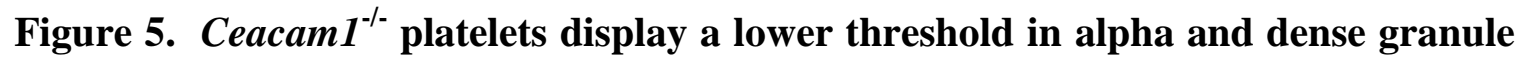
release in response to stimulation with GPVI-selective agonists.

A. Wild-type (black bars), Ceacaml ${ }^{+/}$(gray bars) and Ceacaml ${ }^{-1}$ platelets (white bars) were stimulated with varying concentrations of GPVI-selective agonists including type I fibrillar collagen in the absence of magnesium and CRP, or with thrombin. The amount of 5-hydroxytryptamine released was measured as described in Materials and Methods. The assays were performed in triplicate, and the results expressed as the mean $\pm \mathrm{SEM}$. These results are representative of three independent experiments. Note that the release of dense granular contents in response to stimulation at subthreshold levels of GPVIselective agonists was elevated in Ceacam ${ }^{-1-}$ platelets compared to the wild-type and Ceacaml $^{+/}$platelets.

B. Surface expression of P-selectin (alpha granule release) was determined for washed platelets stimulated by thrombin, PAR-4 agonist peptide or collagen-related peptide (CRP) at different concentrations and then stained with either a buffer control and FITCP-selectin mAb for both wild-type and Ceacam $1^{-/}$platelets. FITC-labelled samples were analyzed on a FACS Canto analyzer. Results are representative of three independent experiments. 
Figure 6. Thrombi are larger and more stable in Ceacam $^{-/-}$mice both in vitro and in vivo.

A. Images of thrombus formation in response to ferric chloride induced vascular injury was visualized in arterioles of wild-type versus Ceacam $^{-/-}$mice over time. The different lengths of time after ferric chloride application are indicated. Note that Ceacaml ${ }^{-/}$ arterioles formed larger thrombi over time compared to wild-type control arterioles $(n=15)$.

B-D. Quantitative analysis of arterial thrombogenesis of wild-type, Ceacaml ${ }^{+/}$and Ceacaml $^{-/-}$arterioles. Compared to wild-type arterioles, Ceacaml ${ }^{-/-}$arterioles exhibited a significant increase in thrombus area over two minutes $(6212 \pm 268.7$ versus $3294 \pm 223.4$ and $\left.2859 \pm 294 \mu \mathrm{m}^{2} ; * * * \mathrm{P}<0.0001, \mathrm{n}=15\right)$, showed greater stability in thrombi formed $(4.07 \pm 0.37$ versus $2.40 \pm 0.21$ and $1.05 \pm 0.12 ; * * * \mathrm{P}<0.001, \mathrm{n}=15)$ and increased thrombus volume $\left(122400 \pm 6794\right.$ versus $49430 \pm 4602$ and $\left.63680 \pm 5478 \mu \mathrm{m}^{3} ; * * * \mathrm{P}<0.0001, \mathrm{n}=15\right)$. The primary stability of the thrombi was scored from 1 to 10 , with 1 being $0-10 \%$ occupancy and 10 being 91-100\% occupancy (ie. complete vessel occlusion) monitored over time.

E-F. Platelet thrombus formation following inhibition of GPVI using monoclonal antibody JAQ1 administration to Ceacaml $1^{+/+}$and Ceacam1 ${ }^{-/}$mice compared to control IgG treated Ceacaml ${ }^{+/+}$and Ceacaml ${ }^{-/}$or untreated Ceacaml ${ }^{+/+}$and Ceacaml ${ }^{-/}$mice. Mice received either $100 \mu \mathrm{g}$ Control IgG or JAQ1 antibody and were left for 5 days before ferric chloride injury and intravital microscopy. Compared to untreated Ceacam1 ${ }^{-}$ /- or control IgG treated Ceacam1 ${ }^{-/}$arterioles, JAQ1 treated Ceacam1 ${ }^{-/}$arterioles 
exhibited a 3-fold reduction in thrombus volume over two minutes (135500 2137 versus $134000 \pm 1837$ compared to $40400 \pm 1127 \mu \mathrm{m}^{3} ; * * * \mathrm{P}<0.001, \mathrm{n}=10$ arterioles from 3 mice/group) and a 2-fold reduction in stability score over two minutes $(6.150 \pm 0.2115$ versus $5.700 \pm 0.3000$ compared to $3.350 \pm 0.2115 ; * * * \mathrm{P}<0.001, \mathrm{n}=10$ arterioles from 3 mice/group). In contrast, compared to untreated Ceacaml $^{+/+}$or control IgG treated Ceacaml $^{+/+}$arterioles, JAQ1 treated Ceacaml ${ }^{+/+}$arterioles exhibited a 3-fold reduction

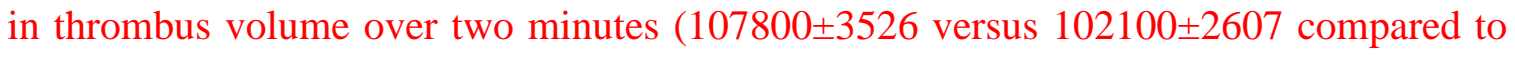
$32050 \pm 599 \mu \mathrm{m}^{3} ; * * * \mathrm{P}<0.001, \mathrm{n}=10$ arterioles from 3 mice/group) and a modest reduction in stability score over two minutes $(2.750 \pm 0.2609$ versus $3.000 \pm 0.2357$ compared to 2.150 $\pm 0.0764 ; * * * \mathrm{P}<0.001, \mathrm{n}=10$ arterioles from 3 mice/group).

G. DiOC $_{6}$-labelled whole blood of wild-type, Ceacam1 $1^{+/}$and Ceacam1 ${ }^{-/}$mice was perfused over $100 \mu \mathrm{g} / \mathrm{mL}$ Type I fibrillar collagen-coated microslides at a wall shear rate of 1800 seconds $^{-1}$. Thrombi were imaged at 4 minutes of blood flow by confocal microscopy (x60, 1- $\mu$ m sections) and thrombus was quantified using Slidebook software (Intelligent Imaging Innovations Inc., Denver, CO, USA). Each data point showed on the graph represents the thrombus volume for each individual mouse performed independently.

Figure 7. Ceacam1 ${ }^{-/-}$mice are more susceptible to Type I collagen-induced pulmonary thromboembolism.

A. The percentage reduction in platelet count over three minutes for wild-type, Ceacaml $^{+/-}$and Ceacaml $^{-/-}$mice after intravenous injection of $350 \mu \mathrm{g} / \mathrm{kg}$ type I fibrillar 
collagen was determined. Note that wild-type, Ceacam1 ${ }^{+/-}$and Ceacaml $^{-/-}$mice showed a similar reduction in platelet count over time $(77.24 \pm 2.246$ versus $73.49 \pm 3.157$ and 74.21 $\pm 3.507 \% ; \mathrm{P}>0.05, \mathrm{n}=20)$.

B. The time until restrained breathing, quantified by a breathing interval of 10 secs, for wild-type and Ceacaml ${ }^{-/}$mice following intravenous injection of $350 \mu \mathrm{g} / \mathrm{kg}$ of type I fibrillar collagen. Note that Ceacaml ${ }^{-/-}$mice showed enhanced susceptibility to type I collagen-induced pulmonary thromboembolism compared to wild-type control mice (343.5 \pm 23.73 versus $327.0 \pm 28.29$ and $252.1 \pm 15.39$ secs; $* * \mathrm{P}<0.001, \mathrm{n}=20)$.

C. The percentage reduction in platelet count over three minutes for wild-type and Ceacaml $^{-/-}$mice after intravenous injection of $0.200 \mu \mathrm{l} / \mathrm{g}$ tissue thromboplastin Thromborel $\mathrm{S}$ was determined. Note that wild-type and Ceacam ${ }^{-/-}$mice showed a similar reduction in platelet count over time $(94.30 \pm 2.162$ versus $93.22 \pm 1.755 \%$; $>>0.05$, $\mathrm{n}=10)$.

D. The time until restrained breathing, quantified by a breathing interval of $10 \mathrm{secs}$, for wild-type and Ceacam $^{-/-}$mice following intravenous injection of $0.200 \mu \mathrm{l} / \mathrm{g}$ tissue thromboplastin Thromborel S was determined. Note that Ceacam $1^{-/-}$mice showed equal susceptibility to tissue thromboplastin induced pulmonary thromboembolism compared to wild-type control mice (214.2 \pm 4.052 versus $223.8 \pm 7.979$ secs; $\mathrm{P}>0.05, \mathrm{n}=10)$. 
Table 1. Summary of peripheral blood haematological parameters for Ceacam ${ }^{- \text {mice }}(\mathrm{n}=18)$.

\begin{tabular}{|c|c|c|c|}
\hline $\begin{array}{l}\text { Haematological } \\
\text { parameter }\end{array}$ & Ceacam $1^{+i+}$ & Ceacamt-1 & $P$ value \\
\hline WCC $\left(\times 10^{9} /\right)$ & $1.63 \pm 0.17$ & $1.81 \pm 0.14$ & $P>0.05$ \\
\hline $\mathrm{Hb}(\mathrm{g} /)$ & $107.8 \pm 2.7$ & $108.3 \pm 3.0$ & $P>0.05$ \\
\hline $\operatorname{RBC}\left(\times 10^{12} \Omega\right)$ & $8.34 \pm 0.148$ & $8.66 \pm 0.230$ & $P>0.05$ \\
\hline $\mathrm{PCV}(/ /)$ & $0.396 \pm 0.007$ & $0.412 \pm 0.009$ & $P>0.05$ \\
\hline $\operatorname{MCV}$ (fl) & $474.8 \pm 1.35$ & $478.1 \pm 2.87$ & $P>0.05$ \\
\hline $\mathrm{MCH}(\mathrm{pg})$ & $129.6 \pm 3.37$ & $125.5 \pm 1.53$ & $P>0.05$ \\
\hline $\mathrm{MCHC}\left(\times 10^{3} \mathrm{~g} /\right)$ & $2.73 \pm 0.07$ & $2.62 \pm 0.03$ & $P>0.05$ \\
\hline Plat $\left(\times 10^{9} /\right)$ & $776 \pm 39$ & $680 \pm 65$ & $P>0.05$ \\
\hline$\%$ Neut./100 WBC & $9.71 \pm 0.84$ & $13.78 \pm 1.88$ & ${ }^{\star} \mathrm{P}<0.05$ \\
\hline$\%$ Lymph./100 WBC & $83.8 \pm 1.7$ & $78.2 \pm 2.7$ & $P>0.05$ \\
\hline$\%$ Mono $/ 100$ WBC & $6.29 \pm 1.21$ & $7.56 \pm 1.14$ & $P>0.05$ \\
\hline
\end{tabular}

WCC White cell count

$\mathrm{Hb}$ Haemoglobin

RBC Red blood cell count

PCV Packed cell volume

MCV Mean cell volume

$\mathrm{MCH}$ Mean cell haemoglobin

MCHC Mean cell haemoglobin concentration

Plat. Platelet count 
Figure 1.
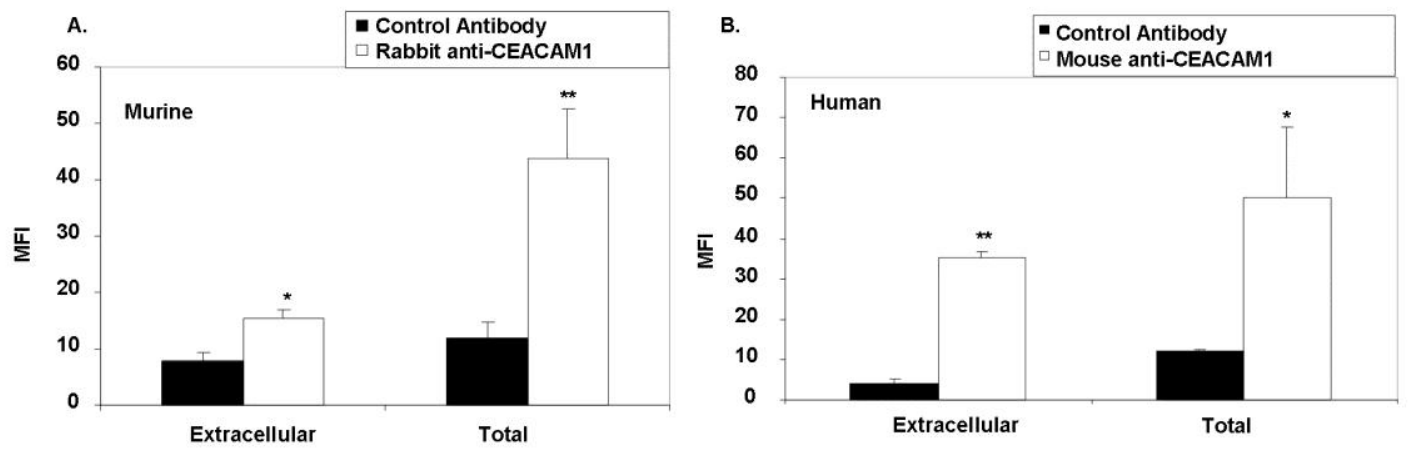

c.

D.
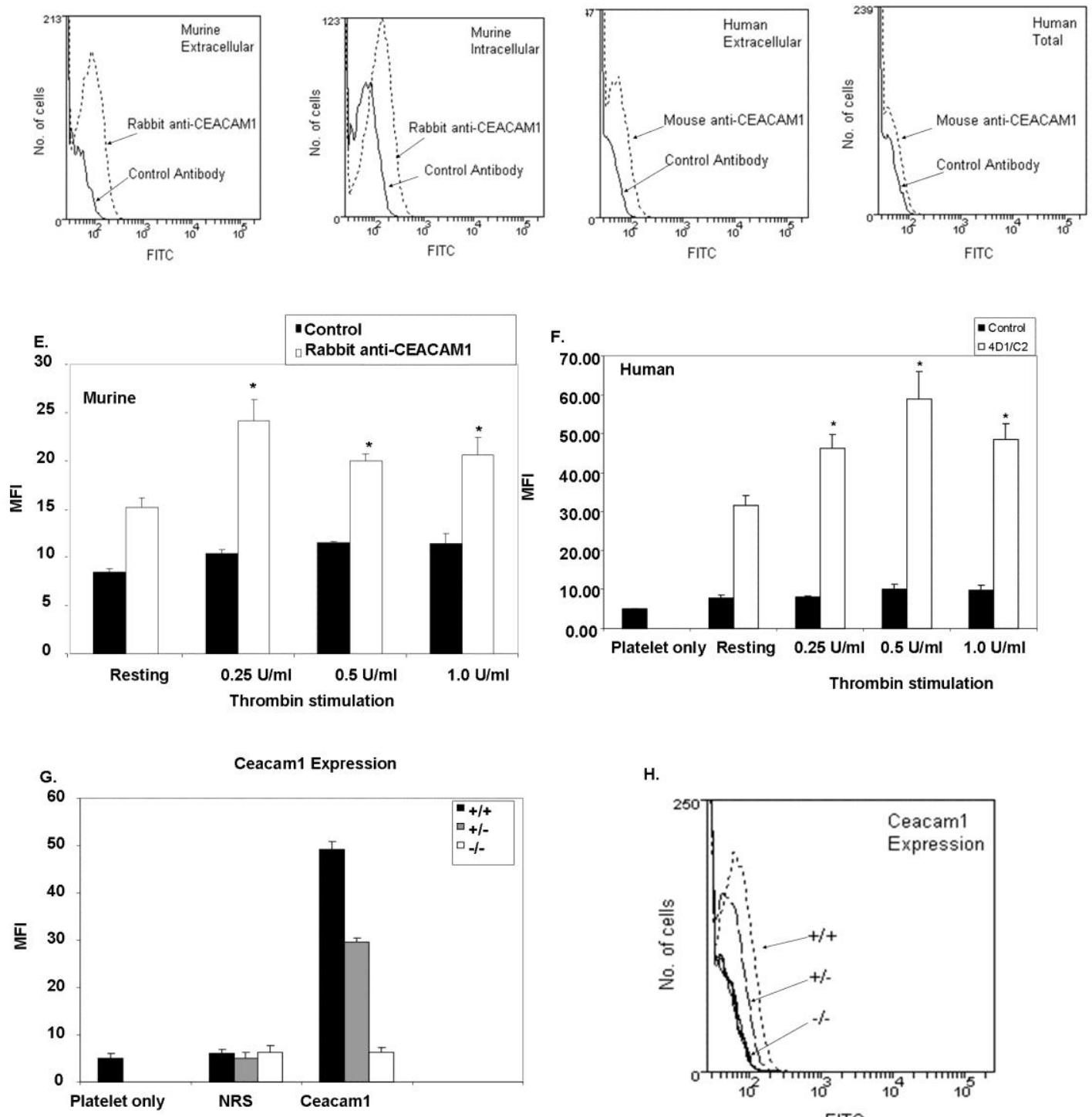

H.

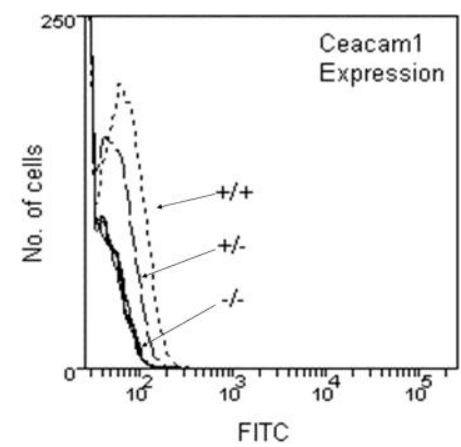


Figure 2.

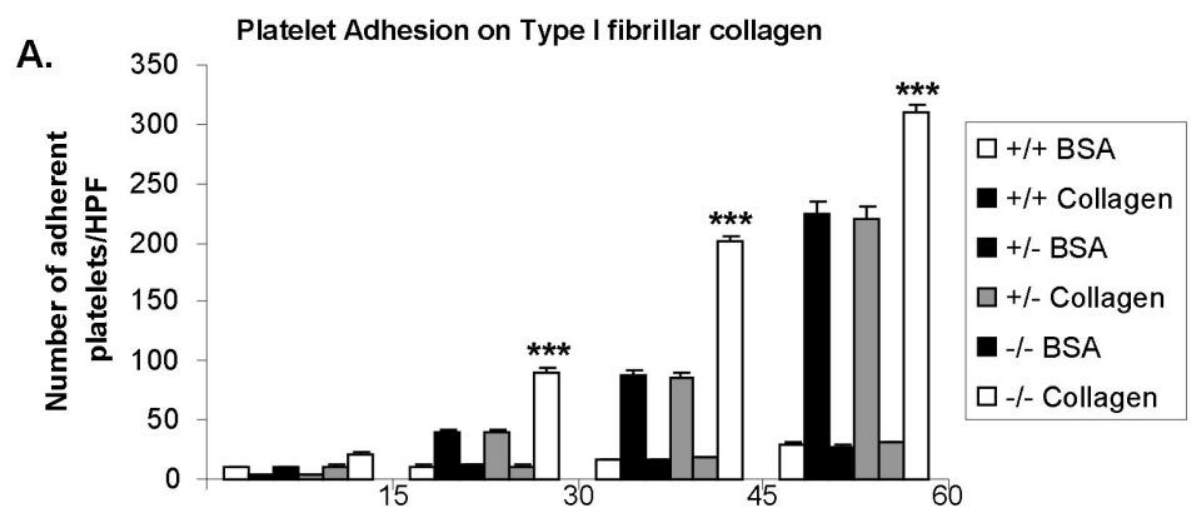

Time (mins)

B.

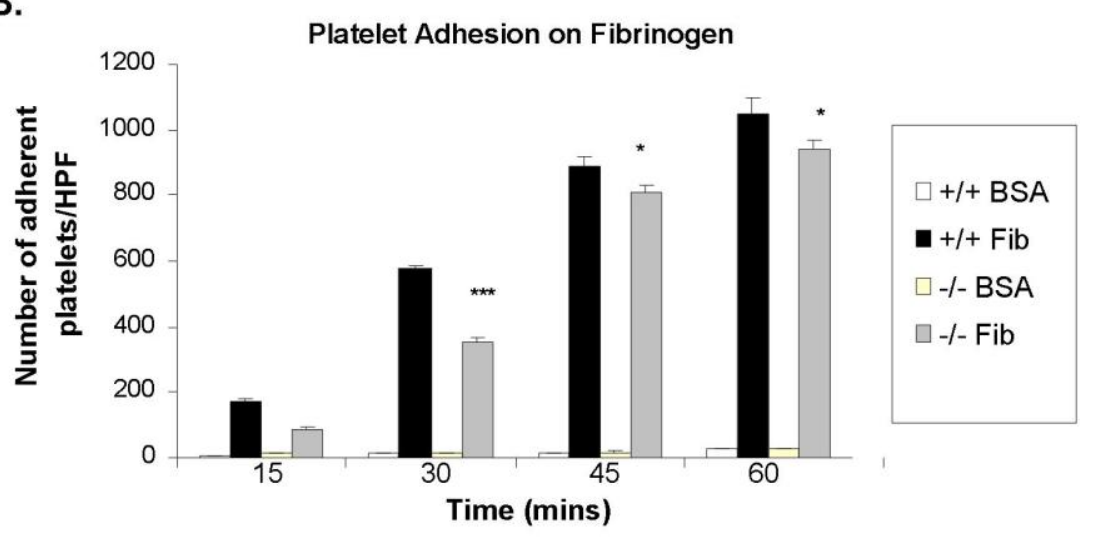

Table 2. Glycoprotein expression in Ceacam1\% mice.

\begin{tabular}{|l|c|c|}
\hline Glycoprotein & $\begin{array}{l}\text { Wild-type mice } \\
\text { (MFI } \pm \text { SEM)(n=3) }\end{array}$ & $\begin{array}{l}\text { Ceacam1 } / \text { mice } \\
\text { (MFI } \pm \text { SEM)(n=3) }\end{array}$ \\
\hline GPVI & $87.9 \pm 5.9$ & $78.3 \pm 9.8$ \\
\hline Integrin $\alpha$ llb $\beta 3$ & $254.7 \pm 15.6$ & $245.0 \pm 16.5$ \\
\hline GPIb $\alpha$ /IXN complex & $242.6 \pm 23.2$ & $231.9 \pm 29.4$ \\
\hline Integrin $\alpha 2 \beta 1$ & $60.7 \pm 1.9$ & $51.9 \pm 2.7$ \\
\hline PECAM-1 & $39.9 \pm 12.7$ & $32.4 \pm 10.1$ \\
\hline CD9 & $258.2 \pm 1.0$ & $256.3 \pm 43.7$ \\
\hline CD44 & $134.7 \pm 2.5$ & $113.3 \pm 10.8$ \\
\hline CEACAM1 & $39.7 \pm 1.4$ & $3.8 \pm 0.04$ \\
\hline
\end{tabular}

Cell surface expression of platelet glycoprotein expression was monitored by flow cytometry using specific monoclonal antibodies for wild-type and ceacam $1^{-\%}$ mice. Mean fluorescence intensity (MFI) were reported with a standard error of mean for three independent experiments. 
Figure 3.

A.

Wild-type

ceacam $1^{-1}$

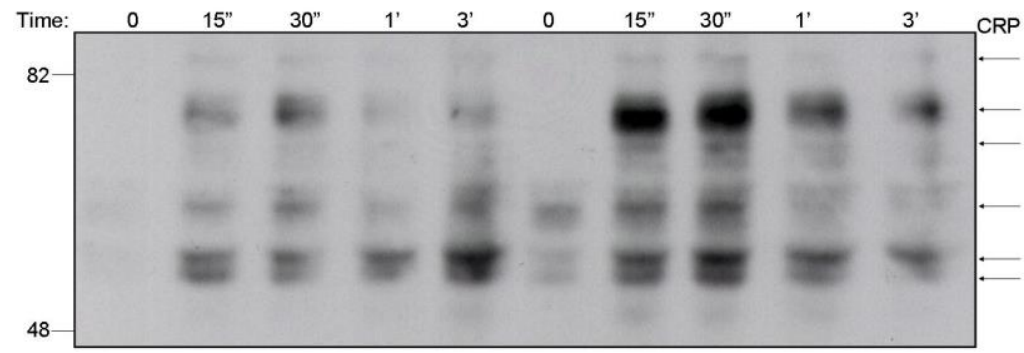

Blot: Anti-phosphotyrosine

48

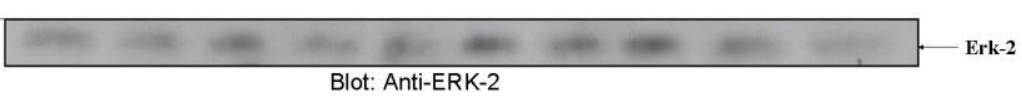

B.
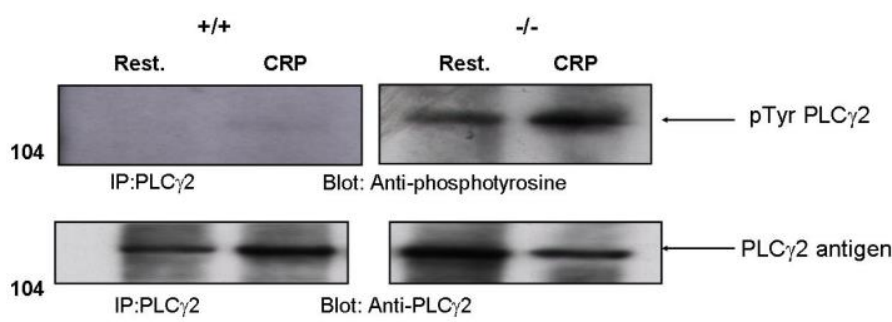

Relative increase

In intensity

of pTyr PLC $\gamma 2$

$8.6 \pm 0.4 \%$

$35.7 \pm 3.2 \%$

$100 \pm 6.4 \%$

to total PLC $\gamma 2$ 
Figure 4.
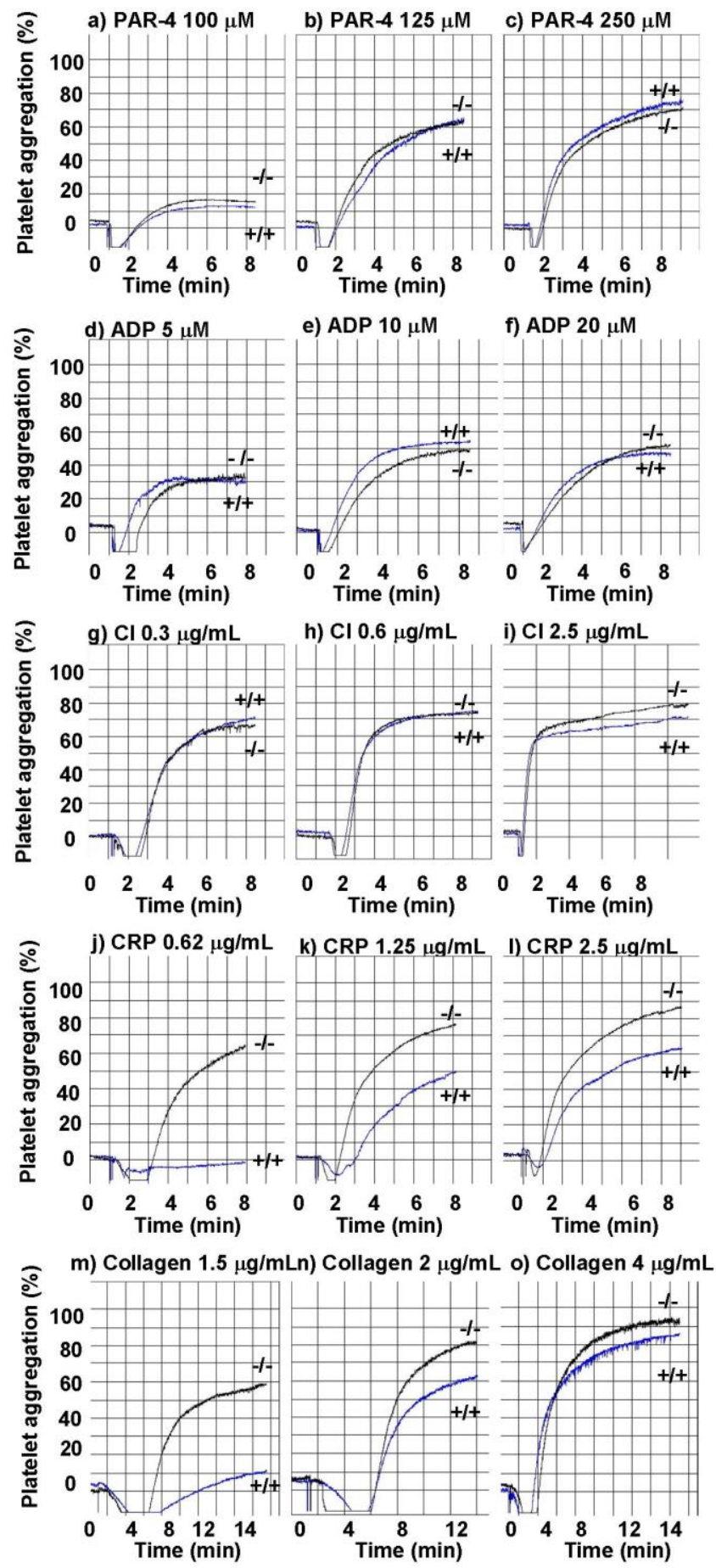
Figure 5.

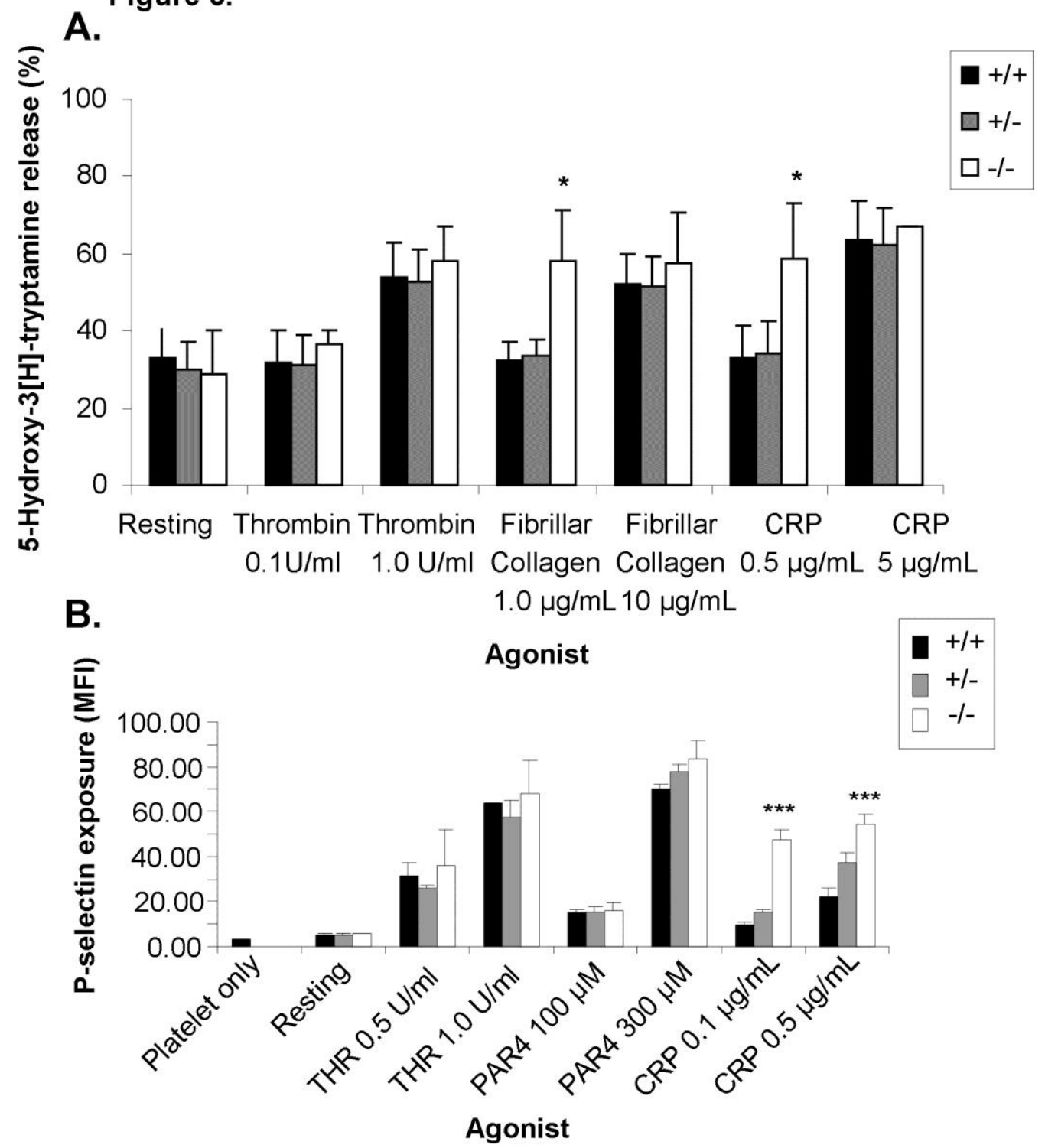


Figure 6.

A.

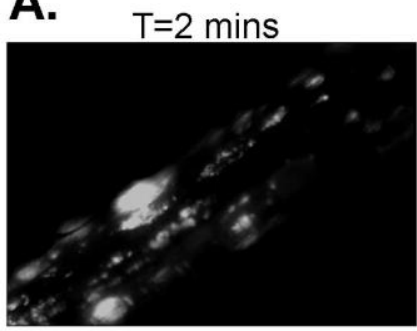

$\mathrm{T}=2 \mathrm{mins}$

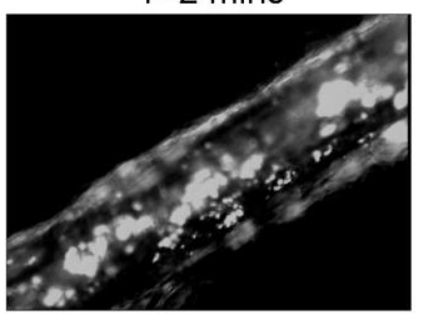

B.

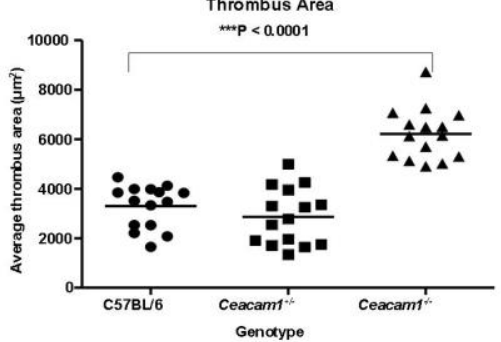

D.
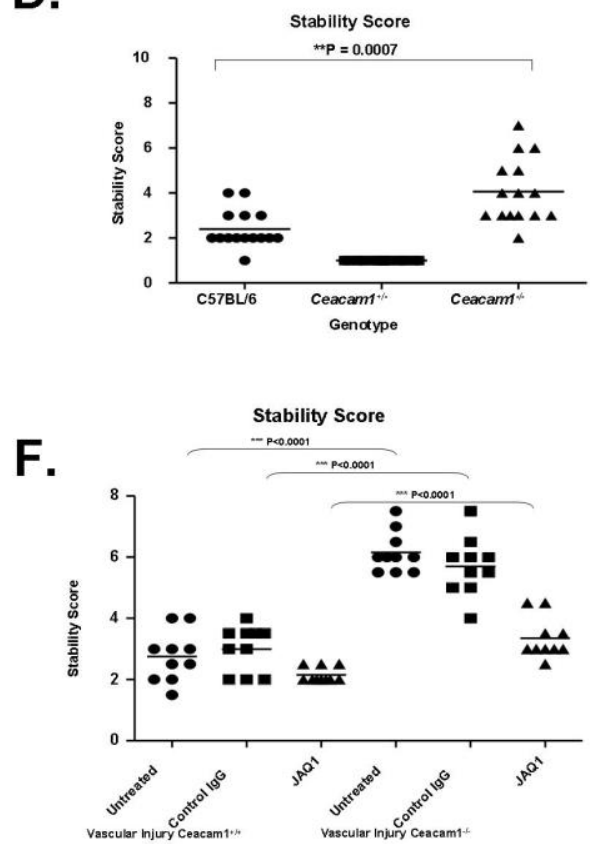

Wild-type C57BL/6
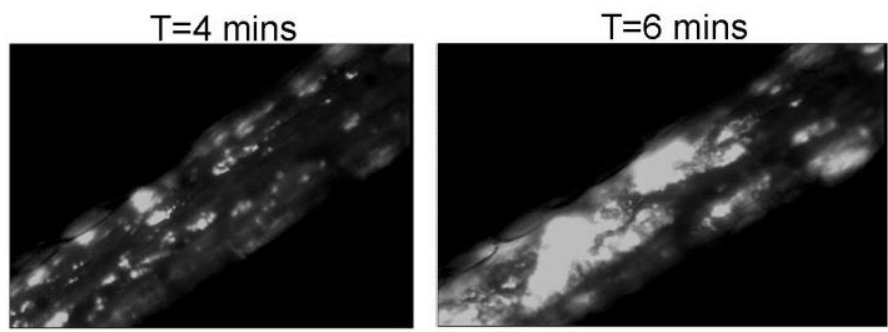

\section{Ceacam 1/}

$\mathrm{T}=4$ mins

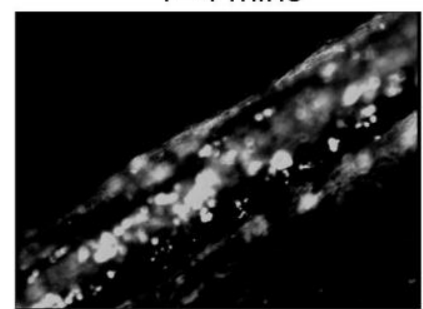

C.
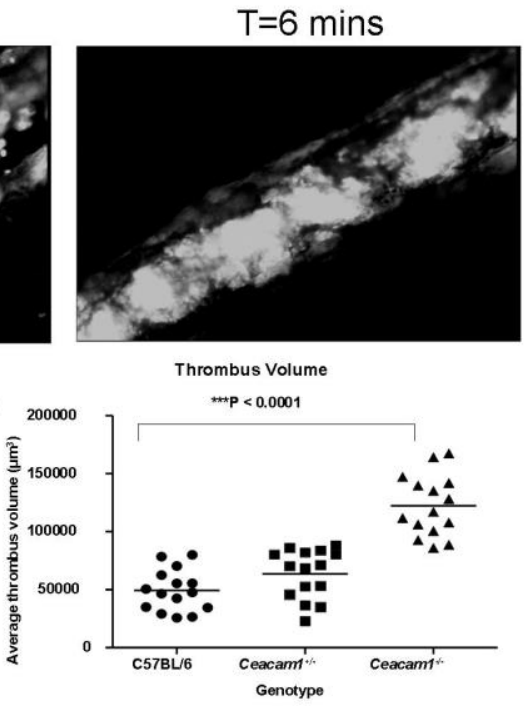

E.

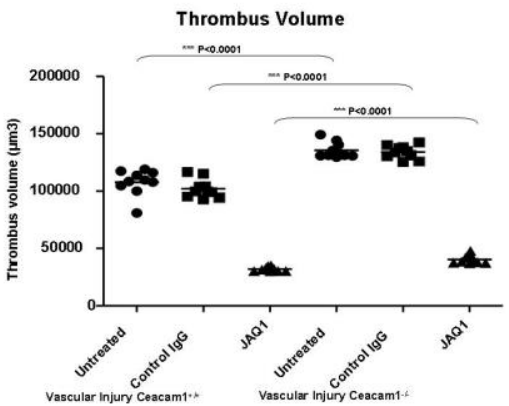

G.

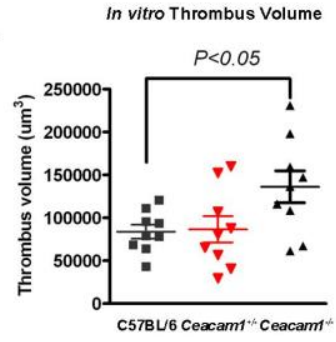


Figure 7.

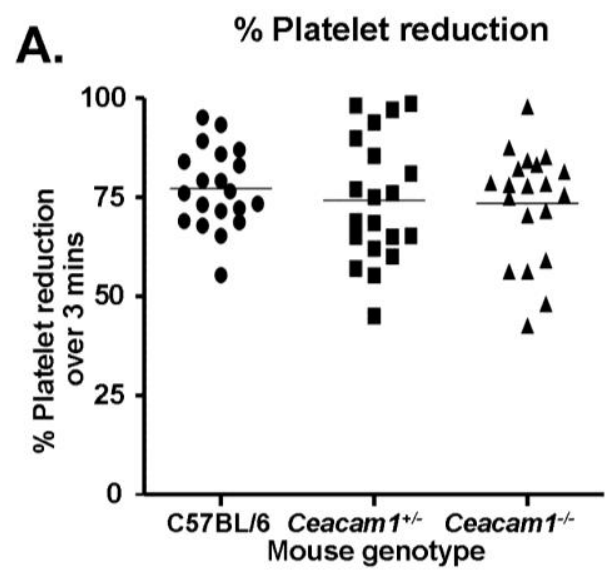

B. Time until restrained breathing
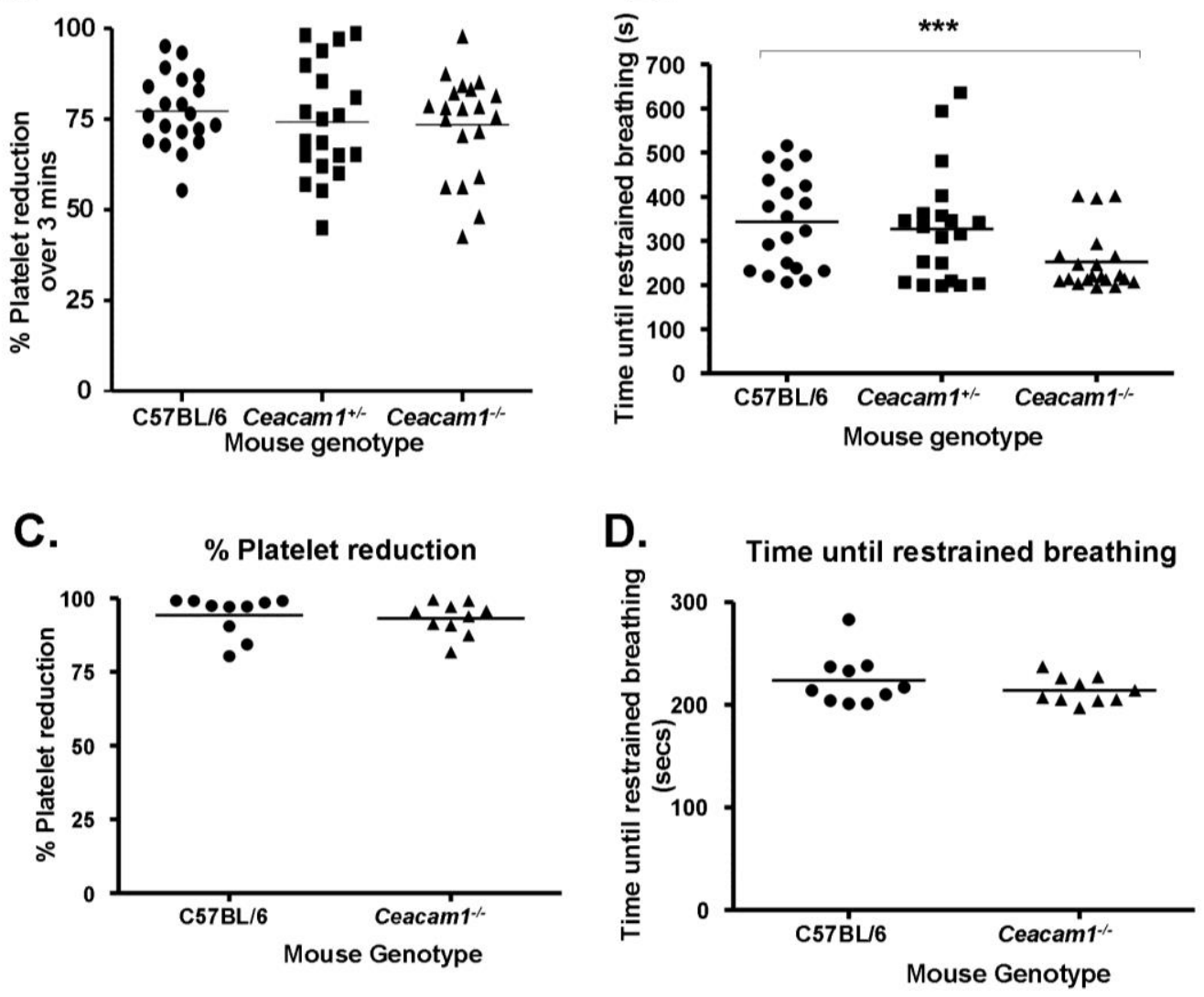